\title{
THE BOLTZMANN EQUATION NEAR A ROTATIONAL LOCAL MAXWELLIAN
}

\author{
CHANWOO KIM AND SEOK-BAE YUN
}

\begin{abstract}
In rotationally symmetric domains, the Boltzmann equation with specular reflection boundary condition has a special type of equilibrium states called the rotational local Maxwellian which, unlike the uniform Maxwellian, has an additional term related to the angular momentum of the gas. In this paper, we consider the initial boundary value problem of the Boltzmann equation near the rotational local Maxwellian. Based on the $L^{2}-L^{\infty}$ framework of [12, we establish the global well-posedness and the convergence toward such equilibrium states.
\end{abstract}

\section{INTRODUCTION}

At the kinetic level, the dynamics of a non-ionized monatomic rarefied gase is governed by the celebrated Boltzmann equation:

$$
\partial_{t} F+v \cdot \nabla_{x} F=Q(F, F) .
$$

Here $F(x, v, t)$ denotes the number density of particles at $(x, v) \in \Omega \times \mathbb{R}^{3}$ in the phase space at time $t$, and $\Omega$ denotes a bounded open subset of $\mathbb{R}^{3}$. The Boltzmann equation describes the evolution of $F$ as a combination of the free transport of particles and the binary collisions. The l.h.s of (1.1) represent the free transport of particles in the absence of collisions while the collision process is encoded in the collision operator $Q$, which takes the following explicit form:

$$
\begin{aligned}
Q\left(F_{1}, F_{2}\right) & =\int_{\mathbb{R}^{3}} \int_{\mathbb{S}^{2}} B(v-u, \omega)\left\{F_{1}\left(v^{\prime}\right) F_{2}\left(u^{\prime}\right)-F_{1}(v) F_{2}(u)\right\} d \omega d u \\
& =Q_{\text {gain }}\left(F_{1}, F_{2}\right)-Q_{\text {loss }}\left(F_{1}, F_{2}\right) .
\end{aligned}
$$

$(u, v)$ and $\left(u^{\prime}, v^{\prime}\right)$ denotes the pre-collisional velocities and the post-collisional velocities respectively and the microscopic conservation laws lead to the following relations between $(v, u)$ and $\left(v^{\prime}, u^{\prime}\right)$, with a free parameter $\omega \in \mathbb{S}^{2}$

$$
u^{\prime}=u+[(v-u) \cdot \omega] \omega, \quad v^{\prime}=v-[(v-u) \cdot \omega] \omega .
$$

We assume that the Boltzmann collision kernel takes the product form as

$$
B(v-u, \omega)=|v-u|^{\gamma} q_{0}(\theta),
$$

where the intermolecular potential is the hard potential $(0 \leq \gamma \leq 1)$ and the collision cross section $q_{0}$ satisfies the Grad cut-off assumption:

$$
0 \leq q_{0}(\theta) \leq C|\cos \theta|, \quad \cos \theta=\frac{(u-v) \cdot \omega}{|u-v|} .
$$

2000 Mathematics Subject Classification. Primary 35Q20, 82C40, 35M13.

Key words and phrases. Kinetic theory, Boltzmann equation, Rotational local Maxwellian, specular reflection boundary condition. 
In this paper, we are interested in the behavior of a rarefied gaseous system contained in a rotationally symmetric domain with specular reflection boundary condition. Due to the fact that the specular reflection, unlike the other boundary conditions, preserves the angular momentum of the particle system, there exists a special type of equilibrium state called the rotational local Maxwellian, which reflect the rotational tendency of the gases. After normalization, it takes the following form:

$$
\mu_{\varpi}(v, x)=\frac{1}{\sqrt{(2 \pi)^{3}}} \exp \left(-\frac{1}{2}|v|^{2}+\varpi \times v \cdot x\right),
$$

where $\varpi$ denotes a vector in $\mathbb{R}^{3}$ related to the angular momentum of the system and the symmetry of the domain. In this paper, we establish the global well-posedness of the initial boundary value problem and prove the convergence toward the rotational local Maxwellian when the initial date is a small perturbation of the rotational local Maxwellian.

The studies of the initial boundary value problem of the Boltzmann equation have rather short history, due mainly to difficulties arising in understanding the complicated interaction between the particles and the boundary, and its ongoing influence on the evolution of the statistical distribution of the gas. Up to now, roughly two types of theories are available for the initial boundary value problem of the Boltzmann equation. The first one is the theory of renormalized solutions by Diperna and Lions introduced in their seminar papers [8, 9]. The extension of this result to the initial boundary value problem was carried out in [18. (See also [1). The advantage of this approach is that the problem can be considered under the most general conditions, namely, the finite mass, energy and entropy of the initial distribution. However, the uniqueness is not guaranteed and remains as one of the most prominent problems in the mathematical kinetic theory.

On the other hand, the semi-group approaches combined with either energy estimate or spectral analysis are also available. The spectral analysis of the linearized collision operator was initiated by Ukai in his pioneering work 34 and its application for the initial boundary value problem can be found in [36, where the external domain problem was considered for specular reflection boundary conditions. The diffusion reflection boundary condition in a bounded domain was studied in [17.

Liu and Yu developed the Green function approach in a series of papers [22, 23, 24, 25]. This approach enables one to obtain the pointwise estimates of the Green function of the linearized Boltzmann equation and get detailed information on how various types of fluidkinetic waves propagate. For this, they introduced two types of decompositions, namely, the long wave-short wave decomposition and the particle-wave decomposition, which are analyzed separately and combined using the mixture lemma.

Recently, a novel $L^{2}-L^{\infty}$ framework has been developed by Guo [12. The name is selfdescriptive: the coercive property of the linearized collision operator is captured in $L^{2}$ space, whereas the weighted $L^{\infty}$ estimate is derived by careful analysis of the iterated Duhamel formula 39 to control the bilinear perturbation. This approach is robust in that all the four most widely used boundary conditions, namely, inflow, bounce-back, specular reflection and diffusive boundary conditions can be treated in a unified framework. It was then employed by the first author to study the formulation and propagation of singularity for the initial boundary value problem for the Boltzmann equation 21 .

Our work is based on the $L^{2}-L^{\infty}$ framework. There are several key difficulties. First, since the linearized collision operator is dependent on spatial variable, it is not clear whether the coercivity property holds uniformly in $x$, which is crucial to obtain the $L^{2}$ decay estimate. It was resolved affirmatively in Lemma 2.4, and then the conservation law of angular momentum combined with the hyperbolic-type energy method developed in [12] gives the 
desired $L^{2}$ estimate. Secondly, due to the complicated form of the compact operator and the multiplicative operator for the rotational local Maxwellian, we should be more careful in studying the velocity decomposition for $L^{\infty}$ estimate. The key observation is that in small velocity regime, where all the microscopic velocities involved are small, the effect of rotation is negligible, and the estimate of the most difficult part can be treated similarly as in the case of the uniform Maxwellian.

Brief overview of the initial boundary value problems for other types of kinetic equations is in order. The initial boundary value problem of the kinetic transport equation was considered in 3. Guo considered the half space problem of the Vlasov Maxwell system in 15, 16. Recently, the specular reflection boundary value problem was resolved by Hwang et al. [19, 20. Similar problem for the Vlasov Poisson equation was studied in the framework of renormalzed solutions was studied in 27. We will not attempt to present a complete set of references for the mathematical theory of the Boltzmann equation. We refer the interested readers to [4, 5, 10, 31, 32, 34, 38, for nice overview of mathematical and physical theory of kinetic equations.

This paper is organized as follows. In section 1, we consider the basic formulation of the problem. In section 2 . we prove preliminary lemmas which plays important roles in the later sections. The section 3 is devoted to $L^{2}$ estimate of the linearized Boltzmann equation capturing the coercivity of the linearized collision operator. In section 4 , using the iterated Duhamel formula, we obtain weighted $L^{\infty}$ estimates which are crucial to control the nonlinear terms. Finally, the main result is proved in section 5. In appendix, we study how the rotational local Maxwellians are derived.

1.1. Domain and characteristics. Let $\Omega$ be a connected and bounded domain. We assume that there exists a smooth function $\xi(x)$ such that $\Omega=\{x: \xi(x)<0\}$. We further assume $\nabla \xi(x) \neq 0$ at the boundary $\partial \Omega=\{x: \xi(x)=0\}$. The outward normal vector at $\partial \Omega$ is given by

$$
n(x)=\frac{\nabla \xi(x)}{|\nabla \xi(x)|}
$$

We say $\Omega$ is real analytic if $\xi$ is real analytic in $x$. We define $\Omega$ as strictly convex if there exists $C_{\xi}>0$ such that

$$
\partial_{i j} \xi(x) \zeta^{i} \zeta^{j} \geq C_{\xi}|\zeta|^{2}
$$

for all $x \in \mathbb{R}^{3}$ such that $\xi(x) \leq 0$, and $\zeta \in \mathbb{R}^{3}$. We say that $\Omega$ is rotationally symmetric around $e_{3}=(0,0,1)$ if for all $x \in \partial \Omega$

$$
x \times e_{3} \cdot n(x)=0 .
$$

Geometric assumption $(\mathcal{A})$ : Throughout this paper, we assume that $\Omega$ satisfies the following geometric conditions:

\section{$(\mathcal{A}): \Omega$ is strictly convex, analytic and rotationally symmetric around $\mathbf{e}_{3}$.}

We denote the phase boundary in the space $\Omega \times \mathbb{R}^{3}$ as $\gamma=\partial \Omega \times \mathbb{R}^{3}$, and split it into an outgoing boundary $\gamma_{+}$, an incoming boundary $\gamma_{-}$, and a singular boundary $\gamma_{0}$ for grazing velocities:

$$
\begin{aligned}
& \gamma_{+}=\left\{(x, v) \in \partial \Omega \times \mathbb{R}^{3}: n(x) \cdot v>0\right\} \\
& \gamma_{-}=\left\{(x, v) \in \partial \Omega \times \mathbb{R}^{3}: n(x) \cdot v<0\right\}
\end{aligned}
$$




$$
\gamma_{0}=\left\{(x, v) \in \partial \Omega \times \mathbb{R}^{3}: n(x) \cdot v=0\right\} .
$$

In terms of $F$, we define the specular boundary condition as

$$
F(t, x, v)=F(t, x, R(x) v), \quad(x, v) \in \gamma^{-},
$$

where

$$
R(x) v=v-2\{n(x) \cdot v\} n(x) .
$$

Given $(t, x, v)$, let $[X(s), V(s)]=[X(s ; t, x, v), V(s ; t, x, v)]=[x+(s-t) v, v]$ be the trajectory (or the characteristics) for the Boltzmann equation (1.1):

$$
\frac{d X(s)}{d s}=V(s), \quad \frac{V(s)}{d s}=0
$$

with the initial condition $[X(t ; t, x, v), V(t ; t, x, v)]=[x, v]$.

Definition 1.1. [12] (Backward exit time) For $(x, v)$ with $x \in \bar{\Omega}$ such that there exists some $\tau>0, x-s v \in \Omega$ for $0 \leq s \leq \tau$, we define $t_{\mathbf{b}}(x, v)>0$ to be the last moment at which the back-time straight line $[X(0 ; t, x, v), V(0 ; t, x, v)]$ remains in the interior of $\Omega$ :

$$
t_{\mathbf{b}}(x, v)=\sup \{\tau>0: x-s v \in \Omega \text { for all } 0 \leq s \leq \tau\}
$$

We also define

$$
x_{\mathbf{b}}(x, v)=x\left(t_{\mathbf{b}}\right)=x-t_{\mathbf{b}} v \in \partial \Omega .
$$

We always have $v \cdot n\left(x_{\mathbf{b}}\right) \leq 0$.

1.2. Boundary conditions and conservation laws. (1.2) in the introduction, we defined the rotational local Maxwellian $\mu_{\varpi}$ as

$$
\mu_{\varpi}(v, x)=\frac{1}{\sqrt{(2 \pi)^{3}}} \exp \left(-\frac{1}{2}|v|^{2}+\varpi \times v \cdot x\right),
$$

where $\varpi$ denotes an fixed vector in $R^{3}$. For notational simplicity, we introduce $m_{\varpi}$ as

$$
m_{\varpi}(x, v) \equiv \frac{1}{\sqrt{(2 \pi)^{3}}} e^{-\frac{|v-\varpi \times x|^{2}}{2}},
$$

satisfying

$$
\mu_{\varpi}(x, v) \equiv e^{\frac{|\varpi \times x|^{2}}{2}} m_{\varpi}(x, v) .
$$

Throughout this paper, we consider the following perturbation:

$$
F=\mu_{\varpi}+\sqrt{m}_{\varpi} f .
$$

Then the Boltzmann equation can be rewritten as

$$
\left\{\partial_{t}+v \cdot \nabla+L^{\varpi}\right\} f=\Gamma^{\varpi}(f, f) . \quad f(0, x, v)=f_{0}(x, v),
$$

with the boundary condition:

$$
f(t, x, v)=f(t, x, R(x) v) \text { for }(x, v) \in \gamma_{-} .
$$

The linear Boltzmann operator is given by

$$
\begin{aligned}
L^{\varpi} f & \equiv-\frac{1}{\sqrt{m_{\varpi}}}\left\{Q\left(\mu_{\varpi}, \sqrt{m_{\varpi}} f\right)+Q\left(\sqrt{m_{\varpi}} f, \mu_{\varpi}\right)\right\} \\
& =\nu^{\varpi} f-K^{\varpi, x} f \\
& =\nu^{\varpi} f-\int K_{w_{\varpi}}^{\varpi, x}\left(v, v^{\prime}\right) f\left(v^{\prime}\right) d v^{\prime}
\end{aligned}
$$


with the collision frequency $\nu^{\varpi}(x, v)=\int_{\mathbb{R}^{3}}|v-u|^{\gamma} q_{0}(\theta) m_{\varpi}(u) d u \sim\left(1+|v-\varpi \times x|^{2}\right)^{\frac{1}{2}}$ for $0 \leq \gamma \leq 1$. For convenience, we define $\mathcal{L}_{\varpi}$ as

$$
\begin{aligned}
\mathcal{L}^{\varpi} f & =-\frac{1}{\sqrt{m_{\varpi}}}\left\{Q\left(m_{\varpi}, \sqrt{m_{\varpi}} f\right)+Q\left(\sqrt{m_{\varpi}} f, m_{\varpi}\right)\right\} \\
& =\nu^{\varpi} f-\int \mathcal{K}^{\varpi, x}\left(v, v^{\prime}\right) f\left(v^{\prime}\right) d v^{\prime} .
\end{aligned}
$$

The following properties of $\mathcal{L}_{\varpi}$ and $L_{\varpi}$ can be readily checked.

$$
\begin{aligned}
L^{\varpi} f & =e^{\frac{|\varpi \times x|^{2}}{2}} \mathcal{L}^{\varpi} f, \\
\nu^{\varpi} & =e^{\frac{|\varpi \times x|^{2}}{2}} \nu^{\varpi}, \\
K^{\varpi, x}\left(v, v^{\prime}\right) & =e^{\frac{|\varpi \times x|^{2}}{2}} \mathcal{K}^{\varpi, x}\left(v, v^{\prime}\right) \\
& =e^{\frac{|\varpi \times x|^{2}}{2}} \mathcal{K}^{0, x}\left(v-\varpi \times x, v^{\prime}-\varpi \times x\right),
\end{aligned}
$$

The kernels of $L$ and $\mathcal{L}$ are given as

$$
\begin{aligned}
& \operatorname{ker} L^{\varpi}=\operatorname{span}\left\{\sqrt{\mu}_{\varpi}, v \sqrt{\mu}_{\varpi},|v|^{2} \sqrt{\mu}_{\varpi}\right\}, \\
& \operatorname{ker} \mathcal{L}^{\varpi}=\operatorname{span}\left\{\sqrt{m}_{\varpi}, v \sqrt{m}_{\varpi},|v|^{2} \sqrt{m}_{\varpi}\right\} .
\end{aligned}
$$

We define the macroscopic projection $P_{\varpi}$ as

$$
P_{\varpi} f=a(x, t) \sqrt{\mu}_{\varpi}+b(x, t) \cdot v \sqrt{\mu}_{\varpi}+c(t, x)|v|^{2} \sqrt{\mu}_{\varpi},
$$

where

$$
a(x, t)=\int_{\mathbb{R}^{3}} f \sqrt{\mu}_{\varpi} d v, \quad b(x, t)=\int_{\mathbb{R}^{3}} f v \sqrt{\mu}_{\varpi} d v, \quad c(t, x)=\int_{\mathbb{R}^{3}} f \sqrt{\mu}_{\varpi}|v|^{2} d v .
$$

The bilinear perturbation is defined as

$$
\begin{aligned}
\Gamma^{\varpi}(f, f) & =\frac{1}{\sqrt{m_{\varpi}}} Q\left(\sqrt{m}_{\varpi} f, \sqrt{m}_{\varpi} f\right) \\
& =\Gamma_{\text {gain }}^{\varpi}(f, f)-\Gamma_{\text {loss }}^{\varpi}(f, f) .
\end{aligned}
$$

With the specular reflection condition (1.6), it is well-known that mass, angular momentum, and energy are conserved for (1.1). Without loss of generality, we may always assume that the mass-angular momentum-energy conservation laws hold for $t \geq 0$, in terms of the perturbation $f:$

$$
\begin{aligned}
& \int_{\Omega \times \mathbb{R}^{3}} f(t, x, v) \sqrt{m}_{\varpi} d x d v=0, \\
& \int_{\Omega \times \mathbb{R}^{3}}(x \times v) f(t, x, v) \sqrt{m}_{\varpi} d x d v=0, \\
& \int_{\Omega \times \mathbb{R}^{3}}|v|^{2} f(t, x, v) \sqrt{m}_{\varpi} d x d v=0 .
\end{aligned}
$$

1.3. Main results. We are now in a place to state our main theorem. We first introduce the weight function for $\beta>\frac{3}{2}$ :

$$
w_{\varpi}(v)=\left(1+|v-\varpi \times x|^{2}\right)^{\beta} .
$$


Theorem 1.2. (1) Assume $\Omega$ satisfies the geometric assumption $(\mathcal{A})$. Then any Maxwellian solution of the Boltzmann equation with the specular boundary condition in $\Omega$ takes the following form after the normalization:

$$
\frac{1}{\sqrt{(2 \pi)^{3}}} \exp \left(-\frac{1}{2}|v|^{2}+\varpi \times v \cdot x\right)
$$

for some fixed vector $\varpi \in \mathbb{R}^{3}$. Moreover, except for the case $\Omega=\mathbb{S}^{3}$, $\varpi$ is given by $\varpi=$ $\left(0,0, \varpi_{3}\right)$ for some $\varpi_{3} \in \mathbb{R}$.

(2) Assume $\Omega$ satisfies the geometric assumption $(\mathcal{A})$ and $\varpi$ is parallel to $e_{3}$. Then there exists $\delta>0$ such that if $F_{0}(x, v)=\mu_{\varpi}+\sqrt{m}_{\varpi} f_{0}(x, v) \geq 0$ and $\left\|w_{\varpi} f_{0}\right\|_{\infty} \leq \delta$, there exists $a$ unique solution $F(t, x, v)=\mu_{\varpi}+\sqrt{m}_{\varpi} f(t, x, v) \geq 0$ to the specular boundary value problem (1.6) to the Boltzmann equation (1.1) such that

$$
\sup _{0 \leq t \leq \infty} e^{\lambda t}\left\|w_{\varpi} f(t)\right\|_{\infty} \leq C\left\|w_{\varpi} f_{0}\right\|_{\infty} .
$$

Moreover, if $f_{0}(x, v)$ is continuous except on $\gamma_{0}$ and

$$
f_{0}(x, v)=f_{0}(x, R(x) v) \quad \text { on } \partial \Omega,
$$

then $f(t, x, v)$ is continuous in $[0, \infty) \times\left\{\bar{\Omega} \times \mathbb{R}^{3} \backslash \gamma_{0}\right\}$.

Remark 1.3. (1) Theorem 1.2 (1) states that the rotational local Maxwellian is essentially the only possible equilibrium state of the Boltzmann equation in a rotationally symmetric domain. The proof will be given in Appendix.

(2) The assumption on $\varpi$ imposed in Theorem $1.2(2)$ is justified by Theorem 1.2 (1), because in the case of $S^{2}$, we may redefine $\varpi$ as $e_{3}$ by the symmetry of $\mathbb{S}^{3}$.

(3) Different proofs of Theorem 1.2 (1) can also be found in [7] and [32] .

1.4. Notations. Before we proceed further, we set some notational conventions for various norms and inner product that will be used in later sections. We define $L^{2}$-norm, $L^{\infty}$-norm and weighted $L^{2}$-norm as:

$$
\begin{aligned}
& \|f\|_{\infty}=e s s \sup |f|, \\
& \|f\|^{2}=\int_{\mathbb{R}^{3}} f^{2}(v) d v, \text { or } \int_{\Omega \times \mathbb{R}^{3}} f^{2}(t, x, v) d x d v, \\
& \|f\|_{v_{\varpi}}^{2}=\int_{\mathbb{R}^{3}} f^{2}(t, x, v)\left(1+|v-\varpi \times x|^{2}\right)^{\frac{1}{2}} d v \text {, or } \\
& \\
& \int_{\Omega \times \mathbb{R}^{3}} f^{2}(t, x, v)\left(1+|v-\varpi \times x|^{2}\right)^{\frac{1}{2}} d x d v .
\end{aligned}
$$

We use the following standard notation for corresponding inner products.

$$
\begin{aligned}
(f, g)_{L_{v}^{2}} & =\int_{\mathbb{R}^{3}} f(v) g(v) d v, \\
(f, g)_{L_{x, v}^{2}} & =\int_{\Omega \times \mathbb{R}^{3}} f(x, v) g(x, v) d x d v, \\
(f, g)_{v_{\varpi}}= & \int_{\mathbb{R}^{3}} f(v) g(v)\left(1+|v-\varpi \times x|^{2}\right)^{\frac{1}{2}} d v, \text { or } \\
& \int_{\Omega \times \mathbb{R}^{3}} f(x, v) g(x, v)\left(1+|v-\varpi \times x|^{2}\right)^{\frac{1}{2}} d x d v .
\end{aligned}
$$




\section{PRELIMINARY}

In this section, we establish important technical lemmas for later use. The following lemma shows that if $\Omega$ satisfies $(\mathcal{A})$, the trajectory cannot reach the singular boundary $\gamma_{0}$ if it is not grazing initially.

Lemma 2.1. (Velocity Lemma) [12] Let $\Omega$ be a domain satisfying the geometric assumption $(\mathcal{A})$. Define the functional along the trajectory as

$$
\alpha(s)=\xi^{2}(X(s))+[V(x) \cdot \nabla \xi(X(s))]^{2}-2\{V(s) \cdot \xi(X(s)) \cdot V(s)\} \xi(X(x)) .
$$

Let $X(s) \in \bar{\Omega}$ for $t_{1} \leq t \leq t_{2}$. Then there exists constant $C_{\xi}$ such that

$$
\begin{gathered}
e^{C_{\xi}\left(\left|V\left(t_{1}\right)\right|+1\right) t_{1}} \alpha\left(t_{1}\right) \leq e^{C_{\xi}\left(\left|V\left(t_{1}\right)\right|+1\right) t_{2}} \alpha\left(t_{2}\right), \\
e^{-C_{\xi}\left(\left|V\left(t_{1}\right)\right|+1\right) t_{1}} \alpha\left(t_{1}\right) \geq e^{-C_{\xi}\left(\left|V\left(t_{1}\right)\right|+1\right) t_{2}} \alpha\left(t_{2}\right) .
\end{gathered}
$$

Proof. See [12].

Lemma 2.2. [12] Let $(t, x, v)$ be connected with $\left(t-t_{b}, x-b_{\mathbf{b}} v, v\right)$ backward in time through a trajectory

(1) The backward exit time $t_{\mathbf{b}}(x, v)$ is lower semicontinuous.

(2) If

$$
v \cdot n\left(x_{\mathbf{b}}\right)<0,
$$

then $\left(t_{\mathbf{b}}(x, v), x_{\mathbf{b}}(x, v)\right)$ are smooth functions of $(x, v)$ so that

$$
\begin{aligned}
& \nabla_{x} t_{\mathbf{b}}=\frac{n\left(x_{\mathbf{b}}\right)}{v \cdot n\left(x_{\mathbf{b}}\right)}, \quad \nabla_{v} t_{\mathbf{b}}=\frac{t_{\mathbf{b}} n\left(x_{\mathbf{b}}\right)}{v \cdot n\left(x_{\mathbf{b}}\right)}, \\
& \nabla_{x} x_{\mathbf{b}}=I+\nabla_{x} t_{\mathbf{b}} \otimes v, \quad \nabla_{v} x_{\mathbf{b}}=t_{\mathbf{b}} I+\nabla_{v} t_{\mathbf{b}} \otimes v .
\end{aligned}
$$

Furthermore, if $\xi$ is real analytic, then $\left(t_{\mathbf{b}}(x, v), x_{\mathbf{b}}(x, v)\right)$ are also real analytic.

(3) Let $x_{i} \in \partial \Omega$, for $i=1,2$ and let $\left(t_{1}, x_{1}, v\right)$ and $\left(t_{2}, x_{2}, v\right)$ be connected with the trajectory. Then there exists a constant $C_{\xi}$ such that

$$
\left|t_{1}-t_{2}\right| \geq \frac{\left|n\left(x_{1}\right) \cdot v\right|}{C_{\xi}|v|^{2}} .
$$

(4) Define the boundary mapping

$$
\Phi_{\mathbf{b}}:(t, x, v) \rightarrow\left(t-t_{\mathbf{b}}, x_{\mathbf{b}}(x, v), v\right) \in \mathbb{R} \times\left\{\gamma_{0} \cup \gamma_{-}\right\}
$$

Then $\Phi_{\mathbf{b}}$ and $\Phi_{\mathbf{b}}^{-1}$ maps zero-measure sets to zero-measure sets between either $\{t\} \times$ $\Omega \times \mathbb{R}^{3}$ and $\mathbb{R} \times\left\{\gamma_{0} \cup \gamma_{-}\right\}$or $\mathbb{R} \times\left\{\gamma_{0} \cup \gamma_{+}\right\} \rightarrow \mathbb{R} \times\left\{\gamma_{0} \cup \gamma_{-}\right\}$.

Proof. See 12

Lemma 2.3. There exists constants $C>0$ such that

$$
K_{\varpi}^{\varpi, x}\left(v, v^{\prime}\right) \leq C\left\{\left|v-v^{\prime}\right|+\left|v-v^{\prime}\right|^{-1}\right\} e^{-\frac{1}{8}\left|v-v^{\prime}\right|^{2}-\frac{1}{8} \frac{\| v-\varpi \times\left. x\right|^{2}-\left.\left|v^{\prime}-\varpi \times x\right|^{2}\right|^{2}}{\left|v-v^{\prime}\right|^{2}}},
$$

and

$$
\begin{aligned}
& \int_{\mathbb{R}^{3}}\left\{\left|v-v^{\prime}\right|+\left|v-v^{\prime}\right|^{-1}\right\} e^{-\frac{1}{8}\left|v-v^{\prime}\right|^{2}-\frac{1}{8} \frac{\| v-\varpi \times\left. x\right|^{2}-\left.\left|v^{\prime}-\varpi \times x\right|^{2}\right|^{2}}{\left|v-v^{\prime}\right|^{2}}} \frac{w_{\varpi}(v)}{w_{\varpi}\left(v^{\prime}\right)} d v^{\prime} \\
& \leq \frac{C}{(1+|v-\varpi \times x|)} .
\end{aligned}
$$


Proof. We first notice that for some $C_{\rho, \beta}>0$,

$$
\left|\frac{w_{\varpi}(v)}{w_{\varpi}\left(v^{\prime}\right)}\right| \leq C\left\{1+\left|v-v^{\prime}\right|^{2}\right\}^{|\beta|} .
$$

Let $v-v^{\prime}=\eta$ and $v^{\prime}=v-\eta$. We now compute as

$$
\begin{aligned}
-\frac{1}{8} \mid v & -\left.v^{\prime}\right|^{2}-\frac{1}{8} \frac{\| v-\varpi \times\left. x\right|^{2}-\left.\left|v^{\prime}-\varpi \times x\right|^{2}\right|^{2}}{\left|v-v^{\prime}\right|^{2}} \\
& =-\frac{1}{8}|\eta|^{2}-\frac{1}{8} \frac{\|\left.\eta\right|^{2}-\left.2(v-\varpi \times x) \cdot \eta\right|^{2}}{|\eta|^{2}} \\
& =-\frac{1}{4}|\eta|^{2}+\frac{1}{2}(v-\varpi \times x) \cdot \eta-\frac{1}{2} \frac{|(v-\varpi \times x) \cdot \eta|^{2}}{|\eta|^{2}} \\
& =-\frac{1}{16}|\eta|^{2}+\frac{1}{2}(v-\varpi \times x) \cdot \eta-\left\{\frac{3}{16}|\eta|^{2}+\frac{1}{2} \frac{|(v-\varpi \times x) \cdot \eta|^{2}}{|\eta|^{2}}\right\} \\
& \leq-\frac{1}{8}|\eta|^{2}-\frac{1}{2}\left(\sqrt{\frac{3}{2}}-1\right)|(v-\varpi \times x) \cdot \eta| \\
& \leq-C\left\{|\eta|^{2}+|(v-\varpi \times x) \cdot \eta|\right\},
\end{aligned}
$$

for some constant $C>0$. Hence it is sufficient to estimate

$$
I=\int_{\mathbb{R}^{3}}\left\{1+\frac{1}{|\eta|}\right\} e^{-C|\eta|^{2}} e^{-C|(v-\varpi \times x) \cdot \eta|} d \eta .
$$

For $|v-\varpi \times x| \leq 1$, we have

$$
I \leq \int_{\mathbb{R}^{3}}\left\{1+\frac{1}{|\eta|}\right\} e^{-C|\eta|^{2}} d \eta<\infty .
$$

For $|v-\varpi \times x| \geq 1$, we make a change of variable $\eta_{\|}=\left\{\eta \cdot \frac{v-\varpi \times x}{|v-\varpi \times x|}\right\} \frac{v-\varpi \times x}{|v-\varpi \times x|}$, which gives $\eta_{\perp}=\eta-\eta_{\|}$so that $|(v-\varpi \times x) \cdot \eta|=|v-\varpi \times x|\left|\eta_{\|}\right|$and $\left|v-v^{\prime}\right| \geq\left|\eta_{\perp}\right|$.

$$
\begin{aligned}
I & \leq C_{\beta} \int_{\mathbb{R}^{2}}\left(\frac{1}{\left|\eta_{\perp}\right|}+1\right) e^{-\frac{C}{4}|\eta|^{2}}\left\{\int_{0}^{\infty} e^{-C|v-\varpi \times x| \times|v \||} d\left|\eta_{\|}\right|\right\} d \eta_{\perp} \\
& =\frac{C_{\beta}}{|v-\varpi \times x|} \int_{\mathbb{R}^{2}}\left(\frac{1}{\left|\eta_{\perp}\right|}+1\right) e^{-\frac{C}{4}|\eta|^{2}}\left\{\int_{0}^{\infty} e^{-C|y|} d y\right\} d \eta_{\perp} \\
& \leq \frac{C_{\beta}}{|v-\varpi \times x|} .
\end{aligned}
$$

In the last line, we used the change of variable: $y=|v-\varpi \times x| \times\left|\eta_{\|}\right|$. This completes the proof.

The following lemma shows that the coercivity estimate holds uniformly with respect to $\varpi$ and $x$.

Lemma 2.4 (Positivity of $L^{\varpi}$ ). There exists a constant $\delta_{0}>0$ which is independent of $x$ and $\varpi$ such that

$$
\begin{aligned}
\left(L^{\varpi} f, f\right)_{L_{x, v}^{2}} & \geq \delta_{0} \int_{\mathbb{R}^{3}} e^{\frac{|\varpi \times x|^{2}}{2}}\left\|\left(I-P_{\varpi}\right) f\right\|_{\nu_{\varpi}}^{2} d x \\
& \geq \delta_{0}\left\|\left(I-P_{\varpi}\right) f\right\|_{\nu_{\varpi}}^{2} .
\end{aligned}
$$

Proof. We define the shift operator $\tau_{y}$ as

$$
\tau_{y}: f(x) \rightarrow f(x+y) .
$$


Then we have from (1.7) and the Fubini's theorem,

$$
\begin{aligned}
\int \nu^{\varpi} f(v) d v & =\int\left|v-v_{*}\right| e^{-\frac{\left|v_{*}-\varpi \times x\right|^{2}}{4}} f(v) d \omega d v d v_{*} \\
& =\int\left|v-v_{*}\right| e^{-\frac{\left|v_{*}\right|^{2}}{4}} f(v+\varpi \times x) d \omega d v d v_{*} \\
& =\nu^{0} \tau_{\varpi \times x} f(v),
\end{aligned}
$$

and

$$
\int K^{\varpi, x}\left(v, v^{\prime}\right) f(v) f\left(v^{\prime}\right) d v d v^{\prime}=\int K^{0, x}\left(v, v^{\prime}\right) \tau_{\varpi \times x} f(v) \tau_{\varpi \times x} f\left(v^{\prime}\right) d v d v^{\prime} .
$$

Hence we have

$$
\begin{aligned}
\left(\mathcal{L}_{\varpi} f, f\right)_{L_{v}^{2}} & =-\int \nu^{\varpi} f(v) d v+\int_{R^{6}} K^{\varpi, x}\left(v, v^{\prime}\right) f(v) f\left(v^{\prime}\right) d v d v^{\prime} \\
& =\int\left\{-\nu^{0} \tau_{\varpi \times x} f+\int^{0, x}\left(v, v^{\prime}\right) \tau_{\varpi \times x} f\left(v^{\prime}\right) d v^{\prime}\right\} \tau_{\varpi \times x} f(v) d v \\
& =\left(\mathcal{L}_{0} \tau_{\varpi \times x} f, \tau_{\varpi \times x} f\right)_{L_{v}^{2}} .
\end{aligned}
$$

By the positivity estimate of the standard linearized collision operator (see [10), there exists a constant $\delta_{0}$, independent of $x$ and $\varpi$, such that

$$
\mathcal{L}_{0}\left(\tau_{\varpi \times x} f, \tau_{\varpi \times x} f\right) \geq \delta_{0}\left\|\left(I-P_{0}\right) \tau_{\varpi \times x} f\right\|_{\nu_{0}}^{2} .
$$

On the other hand, the Fubini's theorem gives

$$
\begin{aligned}
\left\|\left(I-P_{0}\right) \tau_{\varpi \times x} f\right\|_{\nu_{0}}^{2} & =\int\left(I-P_{0}\right) f(v+\varpi \times x)\left(1+|v|^{2}\right)^{\frac{1}{2}} d v \\
& =\int\left(I-P_{\varpi}\right) f(v)\left(1+|v-\varpi \times x|^{2}\right)^{\frac{1}{2}} d v \\
& =\left\|\left(I-P_{\varpi \times x}\right) f\right\|_{\nu_{\varpi}}^{2},
\end{aligned}
$$

which yields

$$
\left(\mathcal{L}_{\varpi} f, f\right)_{L_{v}^{2}} \geq \delta_{0}\left\|\left(I-P_{\varpi \times x}\right) f\right\|_{\nu_{\varpi}}^{2} .
$$

We then multiply $e^{\frac{|\omega \times x|^{2}}{2}}$ and integrate with respect to $x$ to see

$$
\begin{aligned}
\left(L^{\varpi} f, f\right)_{L_{x, v}^{2}} & =\int e^{\frac{|\varpi x x|^{2}}{2}}\left(\mathcal{L}_{\varpi} f, f\right)_{L_{v}^{2}} d x \\
& \geq \delta_{0} \int_{\mathbb{R}^{3}} e^{\frac{|\varpi \times x|^{2}}{2}}\left\|\left(I-P_{\varpi}\right) f\right\|_{\nu_{\varpi}}^{2} d x \\
& \geq \delta_{0}\left\|\left(I-P_{\varpi}\right) f\right\|_{\nu_{\varpi}}^{2} .
\end{aligned}
$$

This completes the proof.

Lemma 2.5. Recall (1.9) and (1.11). We have

$$
\left|w_{\varpi} \Gamma\left(g_{1}, g_{2}\right)(v)\right| \leq C(|v-\varpi \times x|+1)^{\gamma}\left\|w_{\varpi} g_{1}\right\|_{\infty}\left\|w_{\varpi} g_{2}\right\|_{\infty} .
$$

Proof. First consider the second term $\Gamma_{\text {loss }}$. We have

$$
\int_{\mathbb{R}^{3}}|u-v|^{\gamma}\left|\sqrt{m}_{\varpi} g_{2}(x, u)\right| d u \leq C\{|v-\varpi \times x|+1\}^{\gamma}\left\|w g_{2}\right\|_{\infty} .
$$

Hence $w \Gamma_{\text {loss }}\left[g_{1}, g_{2}\right]$ is bounded by

$$
w_{\varpi}\left|g_{1}\right| \int_{\mathbb{R}^{3}}|u-v|^{\gamma}\left|\sqrt{m}_{\varpi} g_{2}(x, u)\right| d u \leq C w_{\varpi}(v)\{|v-\varpi \times x|+1\}^{\gamma}\left\|w_{\varpi} g_{1}\right\|_{\infty} .
$$


For $\Gamma_{\text {gain }}$, by $\left|u^{\prime}\right|^{2}+\left|v^{\prime}\right|^{2}=|u|^{2}+|v|^{2}, w_{\varpi}(v) \leq C w_{\varpi}\left(v^{\prime}\right) w_{\varpi}\left(u^{\prime}\right)$, and

$$
\begin{aligned}
& \int q_{0}(\theta)|u|^{\gamma} e^{-\frac{1}{4}|(v-\varpi \times x) u|^{2}} w_{\varpi}(v)\left|g_{1}\left(u^{\prime}\right)\right|\left|g_{2}\left(v^{\prime}\right)\right| d \omega d u \\
& \quad \leq C \int q_{0}(\theta)|u|^{\gamma} e^{-\frac{1}{4}|(v-\varpi \times x)-u|^{2}} w_{\varpi}\left(v^{\prime}\right) w_{\varpi}\left(u^{\prime}\right)\left|g_{1}\left(u^{\prime}\right)\right|\left|g_{2}\left(v^{\prime}\right)\right| d \omega d u \\
& \quad \leq C\left\|w_{\varpi} g_{1}\right\|_{\infty}\left\|w_{\varpi} g_{2}\right\|_{\infty} \int|u|^{\gamma} e^{-\frac{|u-\varpi \times x-v|^{2}}{2}} d u \\
& \leq C(|v-\varpi \times x|+1)^{\gamma}\left\|w_{\varpi} g_{1}\right\|_{\infty}\left\|w_{\varpi} g_{2}\right\|_{\infty} .
\end{aligned}
$$

This completes the proof.

\section{3. $L^{2}$ DECAY THEORY}

In this section, we study the $L^{2}$ estimate of the linear Boltzmann equation:

$$
\partial_{t} f+v \cdot \nabla_{x} f+L^{\varpi} f=0, \quad f(0, x, v)=f_{0}(x, v) .
$$

We define the boundary integration for $g(x, v), x \in \partial \Omega$

$$
\int_{\gamma_{ \pm}} g d \gamma=\int_{ \pm v \cdot n(x)>0}|n(x) \cdot v| d S_{x} d v
$$

where $d S_{x}$ is the standard surface measure on $\partial \Omega$. We also define

$$
\|h\|_{\gamma}=\|h\|_{\gamma_{+}}+\|h\|_{\gamma_{-}}
$$

to be the $L^{2}(\gamma)$ with respect to the measure $|n(x) \cdot v| d S_{x} d v$. Our main theorem of this section is

Theorem 3.1. Suppose $\Omega$ satisfies the assumption $(\mathcal{A})$. Let $f(t, x, v) \in L^{2}$ be the (unique) solution to the linear Boltzmann equation (3.1) with trace $f_{\gamma} \in L_{\text {loc }}^{2}(\gamma)$. We assume the conservation of mass, angular momentum and energy (1.10). Then there exists $\lambda$ and $C>0$ such that

$$
\sup _{0 \leq t \leq \infty}\left\{e^{\lambda t}\|f(t)\|^{2}\right\} \leq 2\|f(0)\|^{2} .
$$

For this, we establish the following proposition

Proposition 3.2. Suppose $\Omega$ satisfies the geometric assumption $(\mathcal{A})$. Then there exists $M>$ 0 such that for any solution $f(t, x, v)$ to the linearized Boltzmann equation (3.1) satisfying the specular reflection condition (1.6) and the conservation laws of mass, angular momentum and energy (1.10), we have the following estimate:

$$
\int_{0}^{1}\left\|P_{\varpi} f(f)\right\|_{\nu_{\varpi}} \leq \int_{0}^{1}\left\|\left(I-P_{\varpi}\right) f(s)\right\|_{\nu_{\varpi}} d s .
$$

Proof. We first show that Proposition 3.2 implies Theorem 3.1

Proof of Theorem 3.1

(i) The case of $s \in[N, t]$ : We multiply $f$ to (3.1) and integrate on $[N, t]$ to obtain

$$
\|f(t)\|^{2}+2 \int_{N}^{t}\left(L^{\varpi} f, f\right) d s=\|f(N)\|^{2},
$$

which gives from Lemma 2.4

$$
\|f(t)\| \leq\|f(N)\|
$$

(ii) The case of $s \in[0, N]$ : Let $f$ be a solution to (3.1), then $e^{\lambda t} f$ satisfies

$$
\left\{\partial_{t}+v \cdot \nabla_{x}+L\right\}\left\{e^{\lambda t} f\right\}-\lambda e^{\lambda t} f=0 .
$$


We multiply $e^{\lambda t} f$ and integrate over $0 \leq s \leq N$. Then we have from $f_{\gamma} \in L_{l o c}^{2}\left(R_{+} ; L^{2}(\gamma)\right)$

$$
\begin{aligned}
e^{2 \lambda N} & \|f(N)\|^{2}+2 \int_{0}^{N} e^{2 \lambda s}\left(L^{\varpi} f, f\right) d s-\lambda \int_{0}^{N} e^{2 \lambda s}\|f(s)\|^{2} d s \\
& =\|f(0)\|^{2}+\int_{0}^{N} \int_{\gamma_{-}} e^{2 \lambda s} f^{2}(s) d \gamma d s-\int_{0}^{N} \int_{\gamma_{+}} e^{2 \lambda s} f^{2}(s) d \gamma d s \\
& =\|f(0)\|^{2} .
\end{aligned}
$$

In the last line, we used the fact that the total boundary contribution vanishes for specular boundary reflection:

$$
\int_{0}^{N} \int_{\gamma_{-}} e^{2 \lambda s} f^{2}(s) d \gamma d s=\int_{0}^{N} \int_{\gamma_{+}} e^{2 \lambda s} f^{2}(s) d \gamma d s .
$$

Dividing the time interval into $\cup_{k=0}^{N-1}[k, k+1)$ and letting $f_{k}(s, x, v) \equiv f(k+s, x, v)$ for $k=0,1,2, \cdots$, we deduce

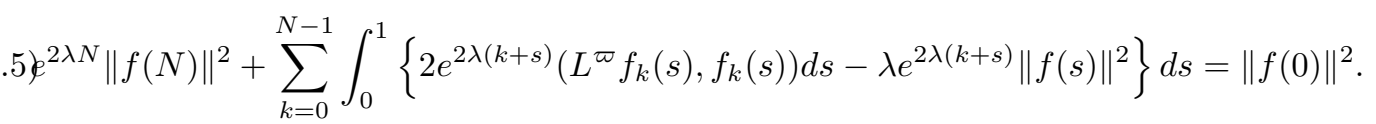

We then apply the positivity estimate in Lemma 2.4 to get

$$
\begin{aligned}
\int_{0}^{1} e^{2 \lambda(k+s)}\left(L^{\varpi} f_{k}(s), f_{k}(s)\right) d s & \geq \delta_{0} \int_{0}^{1} e^{2 \lambda(k+s)}\left\|\left(I-P_{\varpi}\right) f_{k}\right\|_{\nu_{\varpi}}^{2} d s \\
& \geq \delta_{0} \int_{0}^{1} e^{2 \lambda k}\left\|\left(I-P_{\varpi}\right) f_{k}\right\|_{\nu_{\varpi}}^{2} d s .
\end{aligned}
$$

We employ Proposition 3.2 as follows

$$
\begin{aligned}
\int_{0}^{1} e^{2 \lambda k}\left\|\left(I-P_{\varpi}\right) f_{k}\right\|_{\nu_{\varpi}}^{2} d s & =\frac{\delta_{0}}{2} \int_{0}^{1} e^{2 \lambda k}\left\|\left(I-P_{\varpi}\right) f_{k}\right\|_{\nu_{\varpi}}^{2} d s+\frac{\delta_{0}}{2} \int_{0}^{1} e^{2 \lambda k}\left\|\left(I-P_{\varpi}\right) f_{k}\right\|_{\nu_{\varpi}}^{2} d s \\
& \geq \frac{\delta_{0}}{2} \int_{0}^{1} e^{2 \lambda k}\left\|\left(I-P_{\varpi}\right) f_{k}\right\|_{\nu_{\varpi}}^{2} d s+\frac{\delta_{0}}{2 M} \int_{0}^{1} e^{2 \lambda k}\left\|P_{\varpi} f_{k}\right\|_{\nu_{\varpi}}^{2} d s \\
& \geq \frac{1}{2} \min \left\{\frac{\delta_{0}}{M}, \delta_{0}\right\} e^{-2 \lambda} \int_{0}^{t} e^{2 \lambda(k+s)}\|f\|_{\nu_{\varpi}}^{2} d s .
\end{aligned}
$$

We substitute this into (3.5) to get

$$
e^{2 \lambda N}\|f(N)\|^{2}+\left\{\frac{1}{2} \min \left\{\frac{\delta_{0}}{M}, \delta_{0}\right\} e^{-2 \lambda}-C_{\nu_{\varpi}} \lambda\right\} \sum_{k=0}^{N-1} \int_{0}^{1} e^{2 \lambda(k+s)}\|f\|_{\nu_{\varpi}}^{2}=\|f(0)\|^{2} .
$$

Here we have used $\|\cdot\| \leq C_{\nu_{\varpi}}\|\cdot\|_{\nu_{\varpi}}$. We choose $\lambda>0$ small enough such that $\frac{1}{2} \min \left\{\frac{\delta_{0}}{M}, \delta_{0}\right\} e^{-2 \lambda_{-}}$ $C_{\nu_{\varpi}} \lambda>0$, to obtain

$$
e^{2 \lambda N}\|f(N)\|^{2} \leq\|f(0)\|^{2} .
$$

Combining (3.3) of Case i) and (3.6) of Case ii), we have

$$
e^{2 \lambda t}\|f(t)\|^{2} \leq e^{2 \lambda t}\|f(N)\|^{2} \leq e^{2 \lambda(t-N)} e^{2 \lambda N}\|f(t)\|^{2} \leq e^{2 \lambda(t-N)}\|f(0)\|^{2} \leq\|f(0)\|^{2} .
$$

This completes the proof. 
3.1. Proof of Proposition 3.2. We prove Proposition 3.2 by a contradiction argument. For this, we suppose in the contrary that for each $k$, there exists $f_{k}$ which satisfies the conservation laws (1.10), the specular boundary condition (1.6) and

$$
\int_{0}^{1}\left\|P_{\varpi} f_{k}\right\|_{\nu_{\varpi}}^{2} d s \geq k \int_{0}^{1}\left\|\left(I-P_{\varpi}\right) f_{k}\right\|_{\nu_{\varpi}}^{2} d s .
$$

We normalize it as

$$
Z_{k}=\frac{f_{k}}{\sqrt{\int_{0}^{1}\left\|P_{\varpi} f_{k}\right\|_{\nu_{\varpi}}^{2} d s}}
$$

which satisfies

$$
\int_{0}^{1}\left\|P_{\varpi} Z_{k}\right\|_{\nu_{\varpi}}^{2} d s=1
$$

From $\left\{\partial_{t}+v \cdot \nabla+L^{\varpi}\right\} f_{k}=0$, we have

$$
\left\{\partial_{t}+v \cdot \nabla+L^{\varpi}\right\} Z_{k}=\frac{\left\{\partial_{t}+v \cdot \nabla+L^{\varpi}\right\} f_{k}}{\sqrt{\int_{0}^{1}\left\|P_{\varpi} f_{k}\right\|^{2} d s}}=0 .
$$

Dividing both sides of (3.7), we obatin

$$
\int_{0}^{1}\left\|\left(I-P_{\varpi}\right) Z_{k}\right\|_{\nu_{\varpi}}^{2} d s \leq \frac{1}{k} .
$$

From (3.8) and (3.10), there exists $Z(t, x, v)$ such that

$$
Z_{k} \rightarrow Z \text { weakly in } \int_{0}^{1}\|\cdot\|_{\nu_{\varpi}}^{2} d s
$$

and

$$
\int_{0}^{1}\left\|\left(I-P_{\varpi}\right) Z_{k}\right\|_{\nu_{\varpi}}^{2} d s \rightarrow 0 .
$$

On the other hand, it is straightforward to verify

$$
P_{\varpi} Z_{k} \rightarrow P_{\varpi} Z \text { weakly in } \int_{0}^{1}\|\cdot\|_{\nu_{\varpi}}^{2} d s .
$$

Due to (3.11), (3.12) and (3.13), we have

$$
Z(t, v, x)=\left\{a(t, x)+b(t, x) \cdot v+c(t, x)|v|^{2}\right\} \sqrt{m}_{\varpi} .
$$

It also implies, in the sense of distribution,

$$
\partial_{t} Z+v \cdot \nabla Z=0 \text {. }
$$

We will first prove that, using the hyperbolic type energy method developed in [12] that $Z_{k}$ converges strongly to $Z$ in $\int_{0}^{1}\|\cdot\|^{2} d s$, and $\int_{0}^{1}\|Z\|^{2} d s \neq 0$. Then it will be shown that (3.14) and the specular reflection boundary condition imply $Z=0$, which is a contradiction. First, we consider how does $Z$ look like.

Lemma 3.3. [12] There exist constants $a_{0}, c_{1}, c_{2}$, and constant vectors $b_{0}, b_{1}$ and $\varpi$ such that

$$
\begin{aligned}
Z(t, x, v)= & \left(\left\{\frac{c_{0}}{2}|x|^{2}-b_{0} \cdot x+a_{0}\right\}+\left\{-c_{0} t x-c_{1} x+\varpi \times x+b_{0} t+b_{1}\right\} \cdot v\right. \\
& \left.+\left\{\frac{c_{0} t^{2}}{2}+c_{1} t+c_{2}\right\}|v|^{2}\right) \sqrt{m}_{\varpi} .
\end{aligned}
$$


Moreover, these constants are finite:

$$
\left|a_{0}\right|,\left|b_{0}\right|,\left|b_{1}\right|,\left|c_{0}\right|,\left|c_{1}\right|,\left|c_{2}\right|,|\varpi|<\infty .
$$

Proof. See 12.

\subsection{Interior compactness.}

Lemma 3.4. For any smooth function $\mathcal{X}$ such that $\sup \mathcal{X} \in(0,1) \times \Omega$, we have up to a subsequence

$$
\lim _{k \rightarrow \infty} \int_{0}^{1}\left\|\mathcal{X}\left\{Z_{k}-Z\right\}(s)\right\|^{2} d s=0 .
$$

Proof. We multiply the equation (3.9) by $\chi$ to get

$$
\left\{\partial_{t}+v \cdot \nabla_{x}\right\}\left\{\mathcal{X} Z_{k}\right\}=\left\{\left[\partial_{t}+v \cdot \nabla_{x}\right] \mathcal{X}\right\} Z_{k}-\mathcal{X} L^{\varpi} Z_{k} .
$$

We first note that

$$
\begin{aligned}
\left\{\partial_{t}+v \cdot \nabla_{x}\right\}\{\chi\} & \leq(1+|v|)\left(\sup _{[0,1] \times \Omega}\left|\partial_{t} \mathcal{X}\right|+\sup _{[0,1] \times \Omega}\left|\nabla_{x} \mathcal{X}\right|\right) \\
& \leq C_{\mathcal{X}}(1+|v-\varpi \times x|+|\varpi \times x|) \\
& \leq C_{\mathcal{X}, \varpi, \Omega}(1+|v-\varpi \times x|) .
\end{aligned}
$$

Therefore, we have

$$
\int_{0}^{1}\left\|\left\{\left[\partial_{t}+v \cdot \nabla_{x}\right] \mathcal{X}\right\} Z_{k}-\mathcal{X} L^{\varpi} Z_{k}\right\|_{L^{2}}^{2} d s \leq \int_{0}^{1}\left\|Z_{k}\right\|_{\nu_{\varpi}}^{2} d s .
$$

Since $\int_{0}^{1}\left\|Z_{k}(s)\right\|^{2} d s<\infty$, we can deduce from the averaging lemma ([8, 9] $)$ that $\int \xi(t, x) Z_{k}(v) \phi(v) d v$ are compact in $L^{2}([0,1] \times \Omega)$ for any smooth cut-off function $\phi(v)$. It then follows that

$$
\int \mathcal{X} Z_{k}(v)\left[1, v,|v|^{2}\right] \sqrt{m}_{\varpi} d v
$$

are compact in $L^{2}([0,1] \times \Omega)$. Therefore, up to a subsequence, the macroscopic parts of $Z_{k}$ satisfy

$$
\mathcal{X} P_{\varpi} Z_{k} \rightarrow \mathcal{X} P_{\varpi} Z=\mathcal{X} Z \text { strongly in } L^{2}\left([0,1] \times \Omega \times \mathbb{R}^{3}\right) .
$$

Therefore, in light of $\int_{0}^{1}\left\|\left(I-P_{\varpi}\right) Z_{k}(s)\right\|_{\nu_{\varpi}}^{2} \rightarrow 0$ in (3.12), the remaining microscopic parts $\mathcal{X} Z_{k}$ satisfy $\lim _{k \rightarrow \infty} \int_{0}^{1}\left\|\mathcal{X}\left\{I-P_{\varpi}\right\} Z_{k}(s)\right\|^{2} d s=0$, and our lemma follows.

3.3. No time concentration. We first establish $L^{\infty}$ in time estimate for $Z_{k}$ to rule out possible concentration in time, near either $t=0$ or $t=1$.

Lemma 3.5.

$$
\sup _{0 \leq t \leq 1, k \geq 1}\left\|Z_{k}(t)\right\|<\infty
$$

Proof. We first note that $\int_{0}^{1}\left\|f_{k}(s)\right\|_{\gamma}^{2}<\infty$ implies $\int_{0}^{1}\left\|Z_{k}(s)\right\|_{\gamma}^{2}<\infty$. Therefore, by the standard $L^{2}$ estimate for (3.14)

$$
\left\|Z_{k}(t)\right\|^{2}+2 \int_{0}^{t}\left(L^{\varpi} Z_{k}, Z_{k}\right) d s=\left\|Z_{k}(0)\right\|^{2} .
$$

This gives from Lemma 2.4

$$
\left\|Z_{k}(t)\right\| \leq\left\|Z_{k}(0)\right\|
$$


On the other hand, we note that

$$
\int_{0}^{t}\left(L^{\varpi} Z_{k}(t), Z_{k}(t)\right) d t \leq C \int_{0}^{1}\left\|\left\{I-P_{\varpi}\right\} Z_{k}\right\|_{\nu_{\varpi}}^{2} d t \leq \frac{C}{k} .
$$

Therefore, by (3.17) and (3.19)

$$
\begin{aligned}
\left\|Z_{k}(t)\right\|^{2} & =\left\|Z_{k}(0)\right\|^{2}-2 \int_{0}^{t}\left(L^{\varpi} Z_{k}, Z_{k}\right) d s \\
& \geq\left\|Z_{k}(0)\right\|^{2}-\frac{C}{k} .
\end{aligned}
$$

Since $\int_{0}^{1}\left\|Z_{k}(t)\right\|^{2} d t \leq \int_{0}^{t}\left\|Z_{k}(t)\right\|_{\nu_{\varpi}}^{2} \leq C\left\{1+\frac{1}{k}\right\}$ for hard potentials, integrating (3.20) over $0 \leq t \leq 1$ yields

$$
\begin{aligned}
\left\|Z_{k}(0)\right\|^{2} & =\int_{0}^{1}\left\|Z_{k}(t)\right\|^{2} d t+\frac{C}{k} \\
& \leq C \int_{0}^{1}\left\|Z_{k}(t)\right\|_{\nu_{\varpi}}^{2} d t+\frac{C}{k} \\
& \leq C\left\{1+\frac{1}{k}\right\}+\frac{C}{k} .
\end{aligned}
$$

Then the result follows from (3.18) and (3.21).

3.4. No boundary concentration. In this section, we prove that there is no concentration at the boundary $\partial \Omega$. Let

$$
\Omega_{\varepsilon^{4}} \equiv\left\{x \in \Omega: \xi(x)<-\varepsilon^{4}\right\} .
$$

To this end, we will establish a careful energy estimate near the boundary in the thin shelllike region $[0,1] \times\left\{\Omega \backslash \Omega_{\varepsilon^{4}}\right\} \times R^{4}$. For $m>\frac{1}{2}$, for any $(x, v)$, we define the outward moving (inwarding moving) indicator function $\chi_{+}\left(\chi_{-}\right)$as

$$
\begin{aligned}
& \chi_{+}(x, v)=\mathbf{1}_{\Omega \backslash \Omega_{\varepsilon^{4}}}(x) \mathbf{1}_{\left\{|v| \leq \varepsilon^{-m}, n(x) \cdot v>\varepsilon\right\}}(v), \\
& \chi_{-}(x, v)=\mathbf{1}_{\Omega \backslash \Omega_{\varepsilon^{4}}}(x) \mathbf{1}_{\left\{|v| \leq \varepsilon^{-m}, n(x) \cdot v<-\varepsilon\right\}}(v) .
\end{aligned}
$$

The following lemma shows that $Z_{k}$ can be controlled in a shell like region near grazing phase boundary with large velocity.

\section{Lemma 3.6.}

$$
\sup _{k \geq 1} \int_{0}^{1} \int_{\Omega \backslash \Omega_{\varepsilon^{4}}} \int_{\substack{|n(x) \cdot v| \leq \varepsilon,|v| \geq \varepsilon^{-m}}}\left|Z_{k}(s, x, v)\right|^{2} d v d x d s \leq C \varepsilon .
$$

Proof. Recall from (1.8)

$$
P_{\varpi} Z_{k}=\left\{a_{k}(t, x)+v \cdot b_{k}(t, x)+|v|^{2} c_{k}(t, x)\right\} \sqrt{m}_{\varpi} .
$$

We split the estimate and use (3.10) to see

$$
\begin{aligned}
& \int_{0}^{1} \int_{\Omega \backslash \Omega_{\varepsilon^{4}}} \int_{|n(x) \cdot v| \leq \varepsilon,}\left|Z_{k}(s, x, v)\right|^{2} d v d x d s \\
& \quad \leq \int_{0}^{1} \int_{\Omega \backslash \Omega_{\varepsilon^{4}}} \int_{\substack{|n| \geq \varepsilon^{-m} \\
|n(x) \cdot v| \leq \varepsilon,|v| \geq \varepsilon^{-m}}}\left|P_{\varpi} Z_{k}\right|^{2}+\left|\left(I-P_{\varpi}\right) Z_{k}\right|^{2} d v d x d s \\
& \quad \leq \int_{0}^{1} \int_{\Omega \backslash \Omega_{\varepsilon^{4}}} \int_{\substack{|n(x) \cdot v| \leq \varepsilon,|v| \geq \varepsilon^{-m}}}\left|P_{\varpi} Z_{k}(s, x, v)\right|^{2} d v d x d s+\frac{C}{k} .
\end{aligned}
$$


The first term can be bounded by the Fubini Theorem as

$$
\begin{aligned}
& \int_{0}^{1} \int_{\Omega \backslash \Omega_{\varepsilon^{4}}} \int_{\substack{|n(x) \cdot v| \leq \varepsilon,|v| \geq \varepsilon^{-m}}}\left|P_{\varpi} Z_{k}(s, x, v)\right|^{2} d v d x d s \\
& \leq \int_{0}^{1} \int_{\Omega \backslash \Omega_{\varepsilon^{4}}} \int_{\substack{|n(x) \cdot v| \leq \varepsilon \\
|v| \geq \varepsilon^{-m}}}\left|P_{\varpi} Z_{k}(s, x, v)\right|^{2}\left(1+|v-\varpi \times x|^{2}\right)^{\ell} d v d x d s \\
& \leq \int_{0}^{1} \int_{\Omega \backslash \Omega_{\varepsilon^{4}}}\left\{\left|a_{k}(s, x)\right|^{2}+\left|b_{k}(s, x)\right|^{2}+\left|c_{k}(s, x)\right|^{2}\right\} \\
& \times\left\{\int_{\substack{|n(x) \cdot v| \leq \varepsilon,|v| \geq \varepsilon^{-m}}}\left(1+|v-\varpi \times x|^{2}\right)^{\ell} \sqrt{m}_{\varpi} d v\right\} d x d s .
\end{aligned}
$$

We observe that

$$
\begin{aligned}
\left(1+|v-\varpi \times x|^{2}\right)^{\ell} \sqrt{m} \varpi & =\left(1+|v-\varpi \times x|^{2}\right)^{\ell} e^{-\frac{|v-\varpi \times x|^{2}}{4}} \\
& \leq C\left(1+|v|^{2}\right)^{\ell}\left(1+|\varpi|^{2}|x|^{2}\right)^{\ell} e^{-\frac{|v|^{2}}{4}} e^{\frac{|\varpi \times x|^{2}}{8}} \\
& \leq C_{\varpi, \Omega}\left(1+|v|^{2}\right)^{\ell} e^{-\frac{|v|^{2}}{4}} .
\end{aligned}
$$

Therefore we have

$$
\int_{\substack{|n(x) \cdot v| \leq \varepsilon,|v| \geq \varepsilon^{-m}}}\left(1+|v-\varpi \times x|^{2}\right)^{\ell} \sqrt{m_{\varpi}} d v \leq C_{\varpi, \Omega} \int_{\substack{|n(x) \cdot v| \leq \varepsilon,|v| \geq \varepsilon^{-m}}}\left(1+|v|^{2}\right)^{\ell} \sqrt{m_{0}} d v .
$$

By a change of variable $v_{\|}=\{n(x) \cdot v\} n(x)$, and $v_{\perp}=v-v_{\|}$for $|n(x) \cdot v| \leq \varepsilon$, the inner integral is bounded by the sum of

$$
\int_{|n(x) \cdot v| \leq \varepsilon}\left(1+|v|^{2}\right)^{\ell} \sqrt{m_{0}} d v \leq \int_{-\varepsilon}^{\varepsilon} d v_{\|} \int_{R^{2}} e^{-\frac{\left|v_{\perp}\right|^{2}}{8}} d v_{\perp} \leq C \varepsilon
$$

and

$$
\int_{|v| \geq \varepsilon^{-m}}\left\{1+|v|^{2}\right\}^{\ell+2} \sqrt{m_{\varpi}} d v \leq C \varepsilon .
$$

This completes the proof.

We now study the non-grazing parts $\chi_{ \pm} Z_{k}$. For this, we fix $(x, v) \in\left\{\Omega \backslash \Omega_{\varepsilon^{4}} \times \mathbb{R}^{3}\right\}$ and $s \in[\varepsilon, 1-\varepsilon]$. We then define for backward in time $0 \leq t \leq s$

$$
\tilde{\chi}_{+}(t, x, v)=\mathbf{1}_{\Omega \backslash \Omega_{\varepsilon^{4}}}(x-v(t-s)) \mathbf{1}_{\left.|v| \leq \varepsilon^{-m}, n(x-v(t-s)) \cdot v>\varepsilon\right\}}(v),
$$

and forward in time $0 \leq s \leq t$ :

$$
\tilde{\chi}_{-}(t, x, v)=\mathbf{1}_{\Omega \backslash \Omega_{\varepsilon} 4}(x-v(t-s)) \mathbf{1}_{\left\{|v| \leq \varepsilon^{-m}, n(x-(t-s) v) \cdot v<-\varepsilon\right\}}(v) .
$$

Lemma 3.7. (1) For $0 \leq s-\varepsilon^{2} \leq t \leq s$, if $\tilde{\chi}_{+}(t, x, v) \neq 0$ then $n(x) \cdot v>\frac{\varepsilon}{2}>0$. Moreover, $\tilde{\chi}_{+}\left(s-\varepsilon^{2}, x, v\right)=0$, for $x \in \Omega \backslash \Omega_{\varepsilon^{4}}$.

(2) For $s \leq t \leq s+\varepsilon^{2} \leq 1$, if $\tilde{\chi}_{-}(t, x, v) \neq 0$ then $n(x) \cdot v<-\frac{\varepsilon}{2}<0$. Moreover, $\tilde{\chi}_{-}(s+$ $\left.\varepsilon^{2}, x, v\right)=0$, for $x \in \Omega \backslash \Omega_{\varepsilon^{4}}$.

Proof. See [12]

Lemma 3.8. We have the strong convergence

$$
\lim _{k \rightarrow \infty} \int_{0}^{1}\left\|Z_{k}(s)-Z(s)\right\|^{2} d s=0
$$


and $Z(s)$ is not identically zero in the sense that

$$
\int_{0}^{1}\|Z(s)\|_{\nu_{\varpi}}^{2}>0
$$

Moreover, $Z$ satisfies the specular boundary condition:

$$
\left.Z(t, x, v)\right|_{\gamma^{-}}=\left.Z(t, x, R(x) v)\right|_{\gamma^{+}} .
$$

Proof. We multiply $\tilde{\chi}_{ \pm}$with (3.9) to get

$$
\left\{\partial_{t}+v \cdot \nabla_{x}\right\}\left\{\tilde{\chi}_{ \pm} Z_{k}\right\}=-\tilde{\chi}_{ \pm} L^{\varpi} Z_{k} .
$$

Since $\int_{0}^{1}\left\|Z_{k}(t)\right\|_{\gamma}^{2} d s<\infty$, applying the $L^{2}$ estimate backward in time over the shell-like region $\left[s-\varepsilon^{2}, s\right] \times\left[\Omega \backslash \Omega_{\varepsilon^{4}}\right] \times \mathbb{R}^{3}$ for outgoing part $\chi_{+}$, we obtain

$$
\begin{aligned}
& \left\|\chi_{+} Z_{k}(s)\right\|^{2}+\int_{s-\varepsilon^{2}}^{s}\left\|\bar{\chi}_{+} Z_{l}(t)\right\|_{\gamma_{+}}^{2} d t-\int_{s-\varepsilon^{2}}^{s}\left\|\bar{\chi}_{+} Z_{k}(t)\right\|_{\gamma_{+}^{\varepsilon}}^{2} d t \\
& =\left\|\bar{\chi}_{+} Z_{k}\left(s-\varepsilon^{2}\right)\right\|^{2}+\int_{s-\varepsilon^{2}}^{s}\left\|\bar{\chi}_{+} Z_{l}(t)\right\|_{\gamma_{-}}^{2} d t \\
& -\int_{s-\varepsilon^{2}}^{s}\left\|\bar{\chi}_{+} Z_{k}(t)\right\|_{\gamma_{-}^{\varepsilon}}^{2} d t-2 \int_{s-\varepsilon^{2}}^{s}\left(\bar{\chi}_{+} L^{\varpi} Z_{k}, Z_{k}\right)(t) d t,
\end{aligned}
$$

where $\gamma^{\varepsilon} \equiv\left\{x: \chi(x)=-\varepsilon^{4}\right\}$. We observe from Lemma 3.7 that $\bar{\chi}_{+}\left(x, v, s-\varepsilon^{2}\right)=0$ and $\tilde{\chi}_{+}=0$ on $\gamma_{-}$and $\gamma_{-}^{\varepsilon}$, which gives

$$
\left\|\bar{\chi}+Z_{l}\left(s-\varepsilon^{2}\right)\right\|=0
$$

and

$$
\int_{s-\varepsilon^{2}}^{s}\left\|\bar{\chi}_{+} Z_{l}(t)\right\|_{\gamma_{-}}^{2} d t=\int_{s-\varepsilon^{2}}^{s}\left\|\bar{\chi}_{+} Z_{k}(t)\right\|_{\gamma_{-}^{\varepsilon}}^{2} d t=0 .
$$

For $k$ sufficiently large, we use (3.12) to get

$$
\begin{aligned}
\int_{s-\varepsilon^{2}}^{s}\left(\bar{\chi}_{+} L^{\varpi} Z_{k}, Z_{k}\right)(t) d t & \leq \int_{0}^{1} \int_{\Omega \times \mathbb{R}^{3}}\left|L\left\{I-P_{\varpi} Z_{k}(t)\right\} \| Z_{k}(t)\right| d x d v d t \\
& \leq C\left\{\int_{0}^{1} \|\left\{I-P_{\varpi} Z_{k}(t) \|_{\nu_{\varpi}} d t\right\}^{\frac{1}{2}}\left\{\int_{0}^{1}\left\|Z_{k}(t)\right\|_{\nu_{\varpi}}^{2} d t\right\}^{\frac{1}{2}}\right. \\
& \leq \frac{C}{\sqrt{k}} .
\end{aligned}
$$

Therefore, (3.23) reduces to

$$
\left\|\chi_{+} Z_{k}(s)\right\|^{2}+\int_{s-\varepsilon^{2}}^{s}\left\|\bar{\chi}_{+} Z_{k}(t)\right\|_{\gamma_{+}}^{2} d t=\int_{s-\varepsilon^{2}}^{s}\left\|\bar{\chi}_{+} Z_{k}(t)\right\|_{\gamma_{+}^{\varepsilon}}^{2} d t+\frac{C}{\sqrt{k}} .
$$

By the same argument, we can obtain similar estimate for $\tilde{\chi}_{-} Z_{k}$ :

$$
\left\|\chi_{-} Z_{k}(s)\right\|^{2}+\int_{s}^{s+\varepsilon^{2}}\left\|\bar{\chi}_{+} Z_{k}(t)\right\|_{\gamma_{-}}^{2} d t=\int_{s}^{s+\varepsilon^{2}}\left\|\bar{\chi}_{-} Z_{k}(t)\right\|_{\gamma_{-}}^{2} d t+\frac{C}{\sqrt{k}} .
$$

We then combine (3.24) and (3.25) to see

$$
\begin{aligned}
& \int_{\Omega \backslash \Omega_{\varepsilon^{4}}} \int_{\substack{|n(x) \cdot v|>\varepsilon \\
|v| \leq \varepsilon^{-m}}}\left|Z_{k}(s, x, v)\right|^{2} d x d v \\
& =\int\left|\chi_{-} Z_{k}(s)\right|^{2}+\left|\chi_{+} Z_{k}(s)\right|^{2} d x d v
\end{aligned}
$$




$$
\begin{aligned}
& \leq \int_{s}^{s+\varepsilon^{2}}\left\|\bar{\chi}_{-} Z_{k}(t)\right\|_{\gamma_{-}^{\varepsilon}}^{2} d t+\int_{s-\varepsilon^{2}}^{s}\left\|\bar{\chi}_{+} Z_{k}(t)\right\|_{\gamma_{+}^{\varepsilon}}^{2} d t+\frac{C}{\sqrt{k}} \\
& \leq \int_{s-\varepsilon^{2}}^{s+\varepsilon^{2}}\left\|\mathbf{1}_{\left\{|v| \leq \varepsilon^{-m},|n(x) \cdot v| \geq \frac{\varepsilon}{2}\right\}} Z_{k}(t)\right\|_{\gamma^{\varepsilon}}^{2} d t+\frac{C}{\sqrt{k}} .
\end{aligned}
$$

To estimate the first term, we first observe from Lemma 3.3

$$
\left\{\partial_{t}+v \cdot \nabla_{x}\right\}\left\{\mathbf{1}_{|v| \leq \varepsilon^{-m}}\left(Z_{k}-Z\right)\right\}=-\mathbf{1}_{|v| \leq \varepsilon^{-m}} L\left\{I-P_{\varpi}\right\} Z_{k} .
$$

We can apply Ukai's trace theorem (theorem 5.1.1, 35] $)$ to $\left\{1_{|v| \leq \varepsilon^{-m}}\right\}\left(Z_{k}-Z\right)$ over $\bar{\Omega}_{\varepsilon^{4}}$ to get

$$
\begin{aligned}
& \int_{s-\varepsilon^{2}}^{s+\varepsilon^{2}}\left\|\mathbf{1}_{\left\{|v| \leq \varepsilon^{-m},\left|v \cdot n(x) \geq \frac{\varepsilon}{2}\right|\right\}}\left\{Z_{k}(t)-Z(t)\right\}\right\|_{\gamma^{\varepsilon}}^{2} d s \\
& \quad=\int_{s-\varepsilon^{2}}^{s+\varepsilon^{2}}\left\|\mathbf{1}_{\left\{|v| \leq \varepsilon^{-m}\right\}}\left\{\mathbf{1}_{\left\{\left|v \cdot n(x) \geq \frac{\varepsilon}{2}\right|\right\}}\left(Z_{k}(t)-Z(t)\right)\right\}\right\|_{\gamma^{\varepsilon}}^{2} d s \\
& \quad \leq C_{\varepsilon} \int_{\varepsilon}^{1-\varepsilon}\left\|\mathbf{1}_{\left\{|v| \leq \varepsilon^{-m}\right\}}\left\{Z_{k}(t)-Z_{k}(t)\right\}\right\|_{L^{2}\left(\Omega_{\varepsilon^{4}} \times \mathbb{R}^{3}\right)}^{2} d s \\
& \quad+C_{\varepsilon} \int_{\varepsilon}^{1-\varepsilon} \| \mathbf{1}_{\left\{|v| \leq \varepsilon^{-m}\right\}}\left\{\left\{L^{\varpi}\left\{I-P_{\varpi}\right\} Z_{k}(t)\right\} \|_{L^{2}\left(\Omega_{\varepsilon^{4}} \times \mathbb{R}^{3}\right)}^{2} d s\right. \\
& \quad \leq C_{\varepsilon} \int_{\varepsilon}^{1-\varepsilon}\left\|Z_{k}(t)-Z_{k}(t)\right\|_{L^{2}\left(\Omega_{\varepsilon^{4}} \times \mathbb{R}^{3}\right)}^{2} d s+C_{\varepsilon} \int_{\varepsilon}^{1-\varepsilon}\left\|\left\{I-P_{\varpi}\right\} Z_{k}(t)\right\|_{\nu_{\varpi}}^{2} d s \\
& \quad \leq C_{\varepsilon} \int_{\varepsilon}^{1-\varepsilon}\left\|Z_{k}(t)-Z_{k}(t)\right\|_{L^{2}\left(\Omega_{\varepsilon^{4}} \times \mathbb{R}^{3}\right)}^{2} d s+\frac{C_{\varepsilon}}{k}
\end{aligned}
$$

Therefore, for fixed $\varepsilon$, we have from the interior compactness in Lemma 3.4

$$
\lim _{k \rightarrow \infty} \int_{s-\varepsilon^{2}}^{s+\varepsilon^{2}}\left\|\mathbf{1}_{\left\{|v| \leq \varepsilon^{-m},|n(x) \cdot v| \geq \varepsilon\right\}}\left\{Z_{k}(t)-Z(t)\right\}\right\|_{\gamma^{\varepsilon}}^{2} d t=0 .
$$

Hence, for $k$ large, and for any $\varepsilon \leq s \leq 1-\varepsilon$, we have

$$
\begin{aligned}
& \int_{\Omega \backslash \Omega_{\varepsilon^{4}}} \int_{\substack{|v| \leq \varepsilon^{-m} \\
|v \cdot n(x)| \geq \frac{\varepsilon}{2}}}\left|Z_{k}(s, x, v)\right|^{2} d x d v \\
& \leq 2 \int_{s-\varepsilon^{2}}^{s+\varepsilon^{2}}\left\|\mathbf{1}_{\substack{|v| \leq \varepsilon^{-m}|v \cdot n(x)| \geq \frac{\varepsilon}{2} \\
\mid v \cdot(x)}}\left\{Z_{k}(t)-Z_{k}\right\}\right\|_{\gamma^{\varepsilon}}^{2} d t+2 \int_{s-\varepsilon^{2}}^{s+\varepsilon^{2}}\left\|\mathbf{1}_{\substack{|v| \leq \varepsilon^{-m}|v \cdot n(x)| \geq \frac{\varepsilon}{2} \\
\mid \gamma^{-m}}} Z(t)\right\|_{\gamma^{\varepsilon}}^{2} d t+\frac{C}{\sqrt{k}} \\
& \leq \varepsilon+\int_{s-\varepsilon^{2}}^{s+\varepsilon^{2}}\|Z(t)\|_{\gamma^{\varepsilon}}^{2} d s \\
& \leq \varepsilon+2 \varepsilon^{2} \sup _{0 \leq t \leq 1}\|Z(t)\|_{\gamma^{\varepsilon}}^{2} \\
& \leq \varepsilon+C \varepsilon^{2} \text {. }
\end{aligned}
$$

This leads to

$$
\int_{\Omega \backslash \Omega_{\varepsilon^{4}}} \int_{\substack{|v| \leq \varepsilon^{-m},|v \cdot n(x)| \geq \frac{\varepsilon}{2} \\ \mid v(s)}}\left|Z_{k}(s, x, v)\right|^{2} d x d v \leq C \varepsilon .
$$

for sufficiently small $\varepsilon$. In the last line, we used the fact that $Z_{k}$ is smooth due to (3.15) and its trace is given by (3.15) as well. 
Proof of the strong compactness of $Z_{k}$ : By interior compactness, we have

$$
\begin{aligned}
\int_{0}^{1} & \int_{\Omega \times \mathbb{R}^{3}}\left|Z_{k}(s, x, v)-Z(s, x, v)\right|^{2} d x d v d s \\
& =\left(\int_{0}^{\varepsilon}+\int_{\varepsilon}^{1-\varepsilon}+\int_{1-\varepsilon}^{1}\right) \int_{\Omega \times \mathbb{R}^{3}}\left|Z_{k}(s, x, v)-Z(s, x, v)\right|^{2} d x d v d s \\
& \leq C \varepsilon+\int_{\varepsilon}^{1-\varepsilon} \int_{\Omega \times \mathbb{R}^{3}}\left|Z_{k}(s, x, v)-Z(s, x, v)\right|^{2} d x d v d s .
\end{aligned}
$$

We split the main part $\int_{\varepsilon}^{1-\varepsilon}$ as

$$
\begin{aligned}
& \int_{\varepsilon}^{1-\varepsilon} \int_{\Omega \times \mathbb{R}^{3}}\left|Z_{k}(s, x, v)-Z(s, x, v)\right|^{2} d x d v d s
\end{aligned}
$$

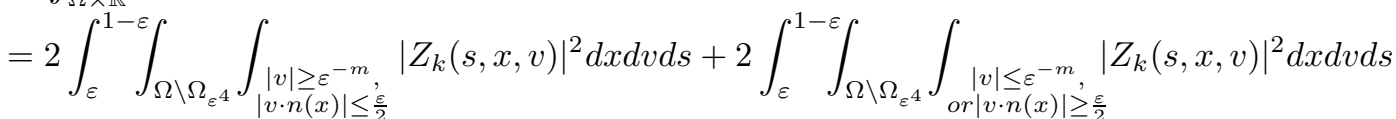

$$
\begin{aligned}
& +2 \int_{\varepsilon}^{1-\varepsilon} \int_{\Omega \backslash \Omega_{\varepsilon}}\left|Z_{k}(s, x, v)\right|^{2} d x d v d s+\int_{\varepsilon}^{1-\varepsilon} \int_{\Omega_{\varepsilon^{4}}}\left|Z_{k}(s, x, v)-Z(s, x, v)\right|^{2} d x d v d s .
\end{aligned}
$$

By (3.27) and Lemma 3.6, the first two terms can be bounded by $C \varepsilon$. The third term is bounded by

$$
\int_{\Omega \backslash \Omega_{\varepsilon^{4}}} \int|Z(t, x, v)|^{2} d v d x \leq C\left|\Omega \backslash \Omega_{\varepsilon^{4}}\right| \leq C \varepsilon .
$$

The last term goes to zero as $k \rightarrow \infty$ by Lemma 3.4. We hence deduce the strong convergence

$$
\int_{0}^{1} \int_{\Omega_{\varepsilon} \times \mathbb{R}^{3}}\left|Z_{k}(s, x, v)-Z(s, x, v)\right|^{2} d x d v d s \rightarrow 0 .
$$

Proof of the positivity of $\mathbf{Z}$ : We first note that both $\int_{0}^{1}\left\|P_{\varpi} Z_{k}\right\|^{2} d t$ and $\int_{0}^{1}\left\|P_{\varpi} Z_{k}\right\|_{\nu_{\varpi}}^{2} d t$ are equivalent to

$$
\int_{0}^{1}\left|a_{k}(s, x)\right|^{2} d x d s+\int_{0}^{1}\left|b_{k}(s, x)\right|^{2} d x d s+\int_{0}^{1}\left|c_{k}(s, x)\right|^{2} d x d s .
$$

Hence we have

$$
\int_{0}^{1}\left\|P_{\varpi} Z_{k}\right\|^{2} d s \geq C \int_{0}^{1}\left\|P_{\varpi} Z_{k}\right\|_{\nu_{\varpi}}^{2} d s=C,
$$

where we used $\int_{0}^{1}\left\|P_{\varpi} Z_{k}(s)\right\|_{\nu_{\varpi}}^{2}=1$. Therefore, we have from the strong convergence of $Z_{k}$ into $Z$ in $\int_{0}^{1}\|\cdot\|^{2} d s$

$$
\int_{0}^{1}\|Z(s)\|^{2} d s=\lim _{k \rightarrow \infty} \int_{0}^{1}\left\|Z_{k}(s)\right\|^{2} d s \geq C>0 .
$$

Boundary condition: Recalling (3.26) and $\int_{0}^{1}\left\|Z_{k}(t)-Z(t)\right\|^{2} d t \rightarrow 0$, we use Ukai's theorem to conclude, for any fixed $\varepsilon>0$,

$$
\begin{aligned}
& \lim _{k \rightarrow \infty} \int_{0}^{1}\left\|\mathbf{1}_{\left\{|v| \leq \varepsilon^{-m},\left|v \cdot n(x) \geq \frac{\varepsilon}{2}\right|\right\}}\left\{Z_{k}(t)-Z(t)\right\}\right\|_{\gamma}^{2} d s \\
& \leq C \lim _{k \rightarrow \infty}\left[\int_{0}^{1}\left\|\mathbf{1}_{\left\{|v| \leq \varepsilon^{-m} \mid\right\}}\left\{Z_{k}(t)-Z(t)\right\}\right\|_{\gamma}^{2} d s\right.
\end{aligned}
$$




$$
\begin{aligned}
& \left.+\int_{0}^{1}\left\|\left[\partial_{t}+v \cdot \nabla_{x}\right] \mathbf{1}_{\left\{|v| \leq \varepsilon^{-m} \mid\right\}}\left\{Z_{k}(t)-Z(t)\right\}\right\|^{2} d s\right] \\
& \leq C \int_{0}^{1}\left\|\mathbf{1}_{\left\{|v| \leq \varepsilon^{-m} \mid\right\}}\left\{L^{\varpi}\left\{I-P_{\varpi}\right\} Z_{k}(t)\right\}\right\|^{2} d s \\
& =0 .
\end{aligned}
$$

$Z_{k}(t, x, v)=Z_{k}(t, x, R(x) v)$ letting $k \rightarrow \infty$, we deduce that $Z$ satisfies $Z(t, x, v)=Z(t, x, R(x) v)$ for $\left\{v \cdot n(x) \geq \frac{\varepsilon}{2}\right\}$. Therefore $Z(t, x, v)=Z(t, x, R(x) v)$ by the continuity of $Z$.

3.5. Boundary condition leads to $Z=0$. We now show that $Z$ has to be zero, which leads to a contradiction. For any fixed $t$, we recall

$$
\left\{\partial_{t}+v \cdot \nabla_{x}\right\}\left\{\mathbf{1}_{|v| \leq \varepsilon^{-m}}\left(Z_{k}-Z\right)\right\}=-\mathbf{1}_{|v| \leq \varepsilon^{-m}} L\left\{I-P_{\varpi}\right\} Z_{k},
$$

and apply Ukai's trace theorem over $[0, t] \times \Omega \times \mathbb{R}^{3}$ to get, for any $0 \leq t \leq 1$,

$$
\lim _{k \rightarrow \infty}\left\|\mathbf{1}_{|v| \leq \varepsilon^{-1}}\left\{Z_{k}(t)-Z(t)\right\}\right\|=0 .
$$

Therefore,

$$
\begin{aligned}
\int Z(t) \sqrt{m}_{\varpi} & =\lim _{k \rightarrow \infty} \int_{|v| \leq \varepsilon^{-1}} Z_{k}(t) \sqrt{m_{\varpi}}+\lim _{k \rightarrow \infty} \int_{|v| \geq \varepsilon^{-1}} Z_{k}(t) \sqrt{m_{\varpi}} \\
\int Z(t)|v|^{2} \sqrt{m} & =\lim _{k \rightarrow \infty} \int_{|v| \leq \varepsilon^{-1}} Z_{k}(t)|v|^{2} \sqrt{m}_{\varpi}+\lim _{k \rightarrow \infty} \int_{|v| \geq \varepsilon^{-1}} Z_{k}(t)|v|^{2} \sqrt{m_{\varpi}}
\end{aligned}
$$

We note that $C\left\|Z_{k}(t)\right\| \int_{|v| \geq \frac{1}{\varepsilon}} m_{\frac{1}{4}}^{\frac{1}{\varpi}}=C \varepsilon$, from the $L^{\infty}$ estimates in Lemma 3.5. This gives

$$
\lim _{k \rightarrow \infty} \int_{|v| \geq \varepsilon^{-1}} Z_{k}(t) \sqrt{m}_{\varpi}=\lim _{k \rightarrow \infty} \int_{|v| \geq \varepsilon^{-1}} Z_{k}(t)|v|^{2} \sqrt{m}_{\varpi}=0 .
$$

We therefore obtain that for all $t$

$$
\begin{array}{r}
\int\left\{\frac{c_{0}}{2}|x|^{2}-b_{0} \cdot x+a_{0}\right\} \sqrt{m}_{\varpi}+\left\{\frac{c_{0} t^{2}}{2}+c_{1} t+c_{2}\right\}|v|^{2} \sqrt{m_{\varpi}} \equiv 0, \\
\int\left\{\frac{c_{0}}{2}|x|^{2}-b_{0} \cdot x+a_{0}\right\}|v|^{2} \sqrt{m}_{\varpi}+\left\{\frac{c_{0} t^{2}}{2}+c_{1} t+c_{2}\right\}|v|^{4} \sqrt{m_{\varpi}} \equiv 0 .
\end{array}
$$

This implies $c_{0}=c_{1}=0$. We therefore have $b=\varpi \times x+b_{0} t+b_{1}$. Now, from the specular reflection, we have for any $x \in \partial \Omega, b(t, x) \cdot n(x)=0$ or

$$
\left\{\varpi \times x+b_{0} t+b_{1}\right\} \cdot n(x) \equiv 0 .
$$

From here, by the same argument employed to derive the rotational local Maxwellian in the Appendix, we arrive at

$$
Z=\varpi \times x \cdot v \sqrt{m}_{\varpi} .
$$

Then, from the conservation of the angular momentum (1.10), we have

$$
\int_{\Omega \times \mathbb{R}^{3}}(x \times v) Z(t) \sqrt{m}_{\varpi} d x d v=\lim _{k \rightarrow \infty} \int_{\Omega \times \mathbb{R}^{3}}(x \times v) Z_{k}(t) \sqrt{m}_{\varpi} d x d v=0 .
$$

Therefore, we combine (3.30) to get

$$
\int_{\Omega \times \mathbb{R}^{3}}(x \times v)(\varpi \times x \cdot v) \sqrt{m}_{\varpi} d x d v=0 .
$$


We now take inner product with $\varpi$ to obtain

$$
0=\varpi \cdot \int_{\Omega \times \mathbb{R}^{3}}(x \times v)(\varpi \times x \cdot v) \sqrt{m}_{\varpi} d x d v=\int\{\varpi \times x \cdot v\}^{2} \sqrt{m}_{\varpi} d v .
$$

Therefore $\varpi=0$ and $Z \equiv 0$.

\section{4. $L^{\infty}$ DECAY THEORY}

In this section, we establish the weighted $L^{\infty}$-estimate which is crucial to control the nonlinear perturbation. For this, we set $h(t, x, v)=w_{\varpi} f(t, x, v)$ and study the equivalent linear Boltzmann equation

$$
\begin{gathered}
\left\{\partial_{t}+v \cdot \nabla_{x}-\nu_{\varpi}+K_{w_{\varpi}, x}^{\varpi}\right\} h=0, \\
h(0, v, x)=h_{0}(x, v) \equiv w_{\varpi} f_{0},
\end{gathered}
$$

where $K_{w_{\varpi}}^{\varpi, x}$ is defined as

$$
\begin{aligned}
K_{w_{\varpi}}^{\varpi, x} h & =w_{\varpi} K^{\varpi, x}\left(\frac{h}{w_{\varpi}}\right) \\
& =\left(1+|v-\varpi \times x|^{2}\right)^{\frac{1}{2}} \int_{\mathbb{R}^{3}} K^{\varpi, x}\left(v, v^{\prime}\right) \frac{h\left(t, x, v^{\prime}\right)}{\left(1+\left|v^{\prime}-\varpi \times x\right|^{2}\right)^{\frac{1}{2}}} d v^{\prime} .
\end{aligned}
$$

Definition 4.1. Let $\Omega$ satisfies the geometric assumption $(\mathcal{A})$. Fix any point $(t, x, v) \in \gamma_{0} \cap \gamma_{-}$ and define $\left(t_{0}, x_{0}, v_{0}\right)=(t, x, v)$ and for $k \geq 1$

$$
\left(t_{k+1}, x_{k+1}, v_{k+1}\right)=\left(t_{k}-t_{\mathbf{b}}\left(t_{k}, x_{k}, v_{k}\right), x_{\mathbf{b}}\left(x_{k}, v_{k}\right), R\left(x_{k+1}\right) v_{k}\right)
$$

And we define the specular back-time cycle

$$
X_{\mathbf{c l}}(s) \equiv \sum_{k=1}^{\infty} \mathbf{1}_{\left[t_{k+1}, t_{k}\right)}(s)\left\{x_{k}+v_{k}\left(s-t_{k}\right)\right\}, \quad V_{\mathbf{c l}}(s) \equiv \sum_{k=1}^{\infty} \mathbf{1}_{\left[t_{k+1}, t_{k}\right)}(s) v_{k} .
$$

Lemma 4.2. Let $\mathcal{M}$ be an operator on $L^{\infty}\left(\gamma_{+}\right) \rightarrow L^{\infty}\left(\gamma_{-}\right)$such that $\|\mathcal{M}\|_{\mathcal{L}\left(L^{\infty}, L^{\infty}\right)}=1$. Then for any $\varepsilon>0$, there exists $h(t) \in L^{\infty}$ and $h_{\gamma} \in L^{\infty}$ solving

$$
\left\{\partial_{t}+v \cdot \nabla_{x}+\nu_{\varpi}\right\} h=0, \quad h_{\gamma_{-}}=(1-\varepsilon) M h_{\gamma_{+}}, \quad h(0, x, v)=h_{0} \in L^{\infty} .
$$

Proof. See 12.

Lemma 4.3. Let $\Omega$ satisfies the geometric assumption $(\mathcal{A})$. Let $h_{0} \in L^{\infty}$ and $G(t) h_{0}$ solves

$$
\left\{\partial_{t}+v \cdot \nabla_{x}+\nu\right\}\left\{G(t) h_{0}\right\}=0,\left\{G(0) h_{0}\right\}=h_{0},
$$

with specular boundary condition $\left\{G(t) h_{0}\right\}(t, x, v)=\left\{G(t) h_{0}\right\}(t, x, R(x) v)$ for $x \in \partial \Omega$. Then for almost all $(x, v) \notin \gamma_{0}$ and $t \in\left[t_{m+1}, t_{m}\right)$

$$
\begin{aligned}
\left\{G(t) h_{0}\right\}(t, x, v) & =e^{-\sum_{k=1}^{m} \nu_{\varpi}\left(x_{k}, v_{k}\right)\left(t_{k}-t_{k+1}\right)} h_{0}\left(X_{\mathbf{c l}}(0), V_{\mathbf{c l}}(0)\right) \\
& =\sum_{k=1}^{\infty} \mathbf{1}_{\left[t_{k+1}, t_{k}\right)}(0) e^{-\sum_{j=1}^{k} \nu_{\varpi}\left(x_{j}, v_{j}\right)\left(t_{j}-t_{j+1}\right)} h_{0}\left(x_{k}-t_{k} v_{k}, v_{k}\right) .
\end{aligned}
$$

Here, we defined $t_{k}=0$ if $t_{k}<0$. Moreover, $e^{\nu_{0} t}\left\|G(t) h_{0}\right\|_{\infty} \leq\left\|h_{0}\right\|_{\infty}$.

Proof. For any $\varepsilon>0$, by Lemma 4.2, there exists a solution $h^{\varepsilon}$ of

$$
\begin{gathered}
\left\{\partial_{t}+v \cdot \nabla_{x}+\nu^{\varpi}(x, v)\right\} h^{\varepsilon}(t, x, v)=0, \\
\left.h^{\varepsilon}(t, x, v)\right|_{\gamma_{-}}=(1-\varepsilon) h^{\varepsilon}(t, x, R(x) v), \\
h^{\varepsilon}(0, x, v)=h_{0} \in L^{\infty} .
\end{gathered}
$$


with $\left\|h^{\varepsilon}(t, \cdot)\right\|_{\infty}<\infty$ and $\sup _{t}\left\|h_{\gamma}^{\varepsilon}(t, \cdot)\right\|_{\infty}<\infty$. Such a solution is necessarily unique. This is because we can choose $w_{\varpi}^{-2}\{1+|v-\varpi \times x|\} \in L^{1}$ so that $f^{\varepsilon}=\frac{h^{\varepsilon}}{w_{\varpi}} \in L^{2}$ is a $L^{2}$ solution to the same equation with the same boundary condition:

$$
\begin{aligned}
& \left\{\partial_{t}+v \cdot \nabla_{x}+\nu^{\varpi}(x, v)\right\} f^{\varepsilon}(t, x, v)=0, \\
& \left.f^{\varepsilon}(t, x, v)\right|_{\gamma_{-}}=(1-\varepsilon) f^{\varepsilon}(t, x, R(x) v), \\
& f^{\varepsilon}(0, x, v)=f_{0} \in L^{\infty} .
\end{aligned}
$$

with an additional property $\int_{0}^{t}\left\|f^{\varepsilon}(s)\right\|_{\gamma}^{2} d s<\infty$. Then uniqueness follows from the energy identity for $f^{\varepsilon}$.

Given any point $(t, x, v) \notin \gamma^{0}$ and its back time cycle $\left[X_{\mathbf{c l}}(s), V_{\mathbf{c l}}(s)\right]$, we notice $\left|V_{\mathbf{c l}}(s)\right|=|v|$ and for $t_{k+1} \leq s \leq t_{k}$,

$$
\begin{aligned}
\varpi \times X_{\mathbf{c l}}(s) \cdot V_{\mathbf{c l}}(s) & =\left(X_{\mathbf{c l}}(s) \times V_{\mathbf{c l}}(s)\right) \cdot \varpi \\
& =\left\{\left(x_{k}+\left(s-t_{k}\right) v_{k}\right) \times v_{k}\right\} \cdot \varpi \\
& =\left(x_{k} \times v_{k}\right) \cdot \varpi \\
& =\varpi \times x_{k} \cdot v_{k} .
\end{aligned}
$$

Therefore, we have $\nu^{\varpi}\left(X_{\mathbf{c l}}(s), V_{\mathbf{c l}}(s)\right)=\nu^{\varpi}\left(x_{k}, v_{k}\right)$ on $\left[t_{k+1}, t_{k}\right]$ and the following identity holds

$$
\frac{d}{d s} G(s) h_{0}=-\nu_{\varpi}\left(x_{k}, v_{k}\right) G(s) h_{0}
$$

along the back time cycle $\left[X_{\mathbf{c l}}(s), V_{\mathbf{c l}}(s)\right]$ for $t_{k+1} \leq s<t_{k}$. Together with the boundary condition at $s=t_{k}$, and part (4) of Lemma 2.2 we deduce that for almost every $(x, v)$, $\frac{d}{d s} G(s) h_{0}$ is constant along its back-time cycle $\left[X_{\mathbf{c l}}(s), V_{\mathbf{c l}}(s)\right]$. If $(x, v) \in \bar{\Omega} \times \mathbb{R}^{3} \backslash \gamma_{0}$, then $t_{\mathbf{b}}(t, v)>0$, and

$$
h^{\varepsilon}(t, x, v)=\sum_{k} \mathbf{1}_{\left[t_{k+1}, t_{k}\right)}(0)(1-\varepsilon)^{k} e^{-\sum_{j=1}^{k} \nu_{\varpi}\left(x_{j}, v_{j}\right)\left(t_{j}-t_{j+1}\right)} h_{0}\left(x_{k}-t_{k} v_{k}, v_{k}\right),
$$

where we put $t_{m}=0$ if $t_{m} \leq 0$.

We now show that for fixed $(t, x, v)$, the number of bounces $k$ is finite. Since $(x, v) \in$ $\bar{\Omega} \times \mathbb{R}^{3} \backslash \gamma_{0}$, by (2.1), we conclude $\alpha(t)>0$, By repeatedly applying velocity Lemma 2.1 along the back-time cycle $\left[X_{\mathbf{c l}}(s), V_{\mathbf{c l}}(s)\right]$, we have for all $k \geq 1$,

$$
e^{-C t_{k}} \alpha\left(t_{k}\right) \geq e^{-C t_{k-1}} \alpha\left(t_{k-1}\right) \geq \ldots \geq e^{-C t} \alpha(t)>0 .
$$

Then, since $\alpha\left(t_{k}\right)=\left\{v_{k} \cdot \nabla \xi\left(x_{k}\right)\right\}^{2}$, we have

$$
\left\{v_{k} \cdot n\left(x_{k}\right)\right\}^{2} \geq C \alpha(t)>0
$$

for all $k \geq 1$, where $C$ depends on $t$ and $v$. Therefore by Lemma 2.2 (3), we have $t_{k}-t_{k-1} \geq$ $\frac{\delta(t)}{C(t, v)|v|^{2}}$. From this we can conclude that the summation over $\mathrm{k}$ is finite.

Now, since $e^{\nu_{0} t}\left\|h^{\varepsilon}\right\|_{\infty}$ and $\sup _{t \geq s, \gamma_{-}}\left|h^{\varepsilon}(t, x, v)\right|$ are uniformly bounded for all $\varepsilon$ :

$$
e^{\nu_{0} t}\left\|h^{\varepsilon}\right\|_{\infty} \leq\left\|h_{0}\right\|_{\infty}, \quad \sup _{t \geq s, \gamma_{-}}\left|h^{\varepsilon}(t, x, v)\right| \leq \sup _{t \geq s, \gamma_{+}}\left|h^{\varepsilon}(t, x, v)\right| \leq\left\|h_{0}\right\|_{\infty},
$$

we can construct the solution $h$ to (4.4) with the original specular reflection boundary condition by taking weak-* limit: $h(t, x, v)=\lim _{\varepsilon} h^{\varepsilon}(t, x, v)$ and $h_{\gamma}(t, x, v)=\lim _{\varepsilon} h_{\gamma}^{\varepsilon}(t, x, v)$. We thus deduce our lemma by letting $\varepsilon \rightarrow 0$. Once again, such a solution $h(t, x, v)$ is necessarily unique in the $L^{\infty}$ class because $f_{\gamma}=\frac{h_{\gamma}}{w_{\varpi}} \in L_{\text {loc }}^{2}\left(L^{2}(\gamma)\right)$. 
Lemma 4.4. Let $\xi$ be convex. Let $h_{0}$ be continuous in $\bar{\Omega} \times \mathbb{R}^{3} \backslash \gamma_{0}$ and $q(t, x, v)$ be continuous in the interior of $[0, \infty) \times \Omega \times \times \mathbb{R}^{3}$ and

$$
\sup _{(0, \infty] \times \Omega \mathbb{R}^{3}}\left|\frac{q(t, x, v)}{\nu^{\varpi}(t, v, x)}\right|<\infty .
$$

Assume that on $\gamma_{-}, h_{0}(x, v)=h_{0}(x, R(x) v)$. Then the specular solution $h(t, x, v)$ to

$$
\left\{\partial_{t}+v \cdot \nabla_{x}+\nu^{\varpi}\right\} h=q, \quad h(0, x, v)=h_{0}(x, v)
$$

is continuous on $[0, \infty) \times\left\{\bar{\Omega} \times \mathbb{R}^{3} \backslash \gamma_{0}\right\}$.

Proof. Take any point $(t, x, v) \notin[0, \infty) \times \gamma_{0}$ and consider its specular back-time $\left[X_{\mathbf{c l}}(s), V_{\mathbf{c l}}(s)\right]$. By repeatedly applying Lemma 2.1 and Lemma 2.2, it follows that $t_{k}(t, x, v), x_{k}(t, x, v)$ and $v_{k}(t, x, v)$ are all smooth functions of $(t, x, v)$. We assume that $t_{m+1} \leq 0<t_{m}$, then $h(t, x, v)$ is given by

$$
\begin{aligned}
h(t, x, v) & =e^{-\mathcal{V}(t, x, v)} h_{0}\left(x_{m}-t_{m} v_{m}, v_{m}\right) \\
& +\sum_{k=0}^{m-1} \int_{t_{k+1}}^{t_{k}} e^{-\mathcal{V}(t-s, x, v)} q\left(s, x_{k}+\left(s-t_{k}\right) v_{k}, v_{k}\right) d s \\
& +\int_{0}^{t_{m}} e^{-\mathcal{V}(t-s, x, v)} q\left(s, x_{m}+\left(s-t_{m}\right) v_{m}, v_{m}\right) d s,
\end{aligned}
$$

where we used the following simplified notation for $t \in\left[t_{k+1}, t_{k}\right]$ :

$$
\begin{aligned}
\mathcal{V}(t, x, v)= & \nu^{\varpi}(x, v)\left(t-t_{1}\right)+\nu^{\varpi}\left(x_{1}, v_{1}\right)\left(t_{1}-t_{2}\right)+ \\
& \cdots+\nu^{\varpi}\left(x_{m-1}, v_{m-1}\right)\left(t_{m-1}-t_{m}\right)+\nu^{\varpi}\left(x_{m}, v_{m}\right) t_{m} .
\end{aligned}
$$

For any point $(\bar{t}, \bar{x}, \bar{v})$ which is close to $(t, x, v)$, we now show that $h(\bar{t}, \bar{x}, \bar{v})$ is close to $h(t, x, v)$ by separating two different cases.

Case I: $t_{m+1}<0$ : or equivalently, $x_{m}+\left(s-t_{m}\right) v_{m} \in \Omega$, away from the boundary. By continuity, $\bar{t}_{m+1}<0$. Therefore, we have

$$
\begin{aligned}
h(\bar{t}, \bar{x}, \bar{v}) & =e^{-\mathcal{V}(\bar{t}, \bar{x}, \bar{v})} h_{0}\left(\bar{x}_{m}-\bar{t}_{m} \bar{v}_{m}, \bar{v}_{m}\right) \\
& +\sum_{k=0}^{m-1} \int_{\bar{t}_{k+1}}^{\bar{t}_{k}} e^{-\mathcal{V}(\bar{t}-s, \bar{x}, \bar{v})} q\left(s, \bar{x}_{k}+\left(s-\bar{t}_{k}\right) \bar{v}_{k}, \bar{v}_{k}\right) d s \\
& +\int_{0}^{\bar{t}_{m}} e^{-\mathcal{V}(\bar{t}-s, \bar{x}, \bar{v})} q\left(s, \bar{x}_{m}+\left(s-\bar{t}_{m}\right) \bar{v}_{m}, \bar{v}_{m}\right) d s,
\end{aligned}
$$

with

$$
\begin{aligned}
\mathcal{V}(\bar{t}, \bar{x}, \bar{v})= & \nu^{\varpi}(\bar{x}, \bar{v})\left(\bar{t}-\bar{t}_{1}\right)+\nu^{\varpi}\left(\bar{x}_{1}, \bar{v}_{1}\right)\left(\bar{t}_{1}-\bar{t}_{2}\right)+ \\
& \cdots+\nu^{\varpi}\left(\bar{x}_{m-1}, \bar{v}_{m-1}\right)\left(\bar{t}_{m-1}-\bar{t}_{m}\right)+\nu^{\varpi}\left(\bar{x}_{m}, \bar{v}_{m}\right) \bar{t}_{m} .
\end{aligned}
$$

Therefore, $h(\bar{t}, \bar{x}, \bar{v}) \rightarrow h(t, x, v)$ follows from the continuity of $\bar{t}_{\ell} \rightarrow t_{\ell}, \bar{x}_{\ell}, \rightarrow x_{\ell}, \bar{v}_{\ell} \rightarrow v_{\ell}$.

Case II: $t_{m+1}=0$ : or equivalently, $x_{m}+\left(s-t_{m}\right) v_{m} \in \partial \Omega$. From (4.5), $\left(x_{k+1} v_{k}\right) \notin \gamma_{0}$. Then by continuity, we know that $\bar{t}_{m}>0$, and $\bar{t}_{m+1}$ is close to zero. However, if $\bar{t}_{m+1}>0$, 
then $\bar{t}_{m+2}<0$, due to $t_{m+2}<t_{m+1}=0$. Therefore $h(\bar{t}, \bar{x}, \bar{v})$ is given by

$$
\begin{aligned}
h(\bar{t}, \bar{x}, \bar{v}) & =e^{-\mathcal{V}(\bar{t}, \bar{x}, \bar{v})} h_{0}\left(\bar{x}_{m+1}-\bar{t}_{m+1} \bar{v}_{m+1}, \bar{v}_{m+1}\right) \\
& +\sum_{k=0}^{m} \int_{\bar{t}_{k+1}}^{\bar{t}_{k}} e^{-\mathcal{V}(\bar{t}-s, \bar{x}, \bar{v})} q\left(s, \bar{x}_{k}+\left(s-\bar{t}_{k}\right) \bar{v}_{k}, \bar{v}_{k}\right) d s \\
& +\int_{0}^{\bar{t}_{m+1}} e^{-\mathcal{V}(\bar{t}-s, \bar{x}, \bar{v})} q\left(s, \bar{x}_{m+1}+\left(s-\bar{t}_{m+1}\right) \bar{v}_{m+1}, \bar{v}_{m+1}\right) d s,
\end{aligned}
$$

with

$$
\begin{aligned}
\mathcal{V}(\bar{t}, \bar{x}, \bar{v})= & \nu^{\varpi}(\bar{x}, \bar{v})\left(\bar{t}-\bar{t}_{1}\right)+\nu^{\varpi}\left(\bar{x}_{1}, \bar{v}_{1}\right)\left(\bar{t}_{1}-\bar{t}_{2}\right)+ \\
& \cdots+\nu^{\varpi}\left(\bar{x}_{m}, \bar{v}_{m}\right)\left(\bar{t}_{m}-\bar{t}_{m+1}\right)+\nu^{\varpi}\left(\bar{x}_{m+1}, \bar{v}_{m+1}\right) \bar{t}_{m+1} .
\end{aligned}
$$

We notice that since $\bar{t}_{m+1} \rightarrow 0$, the $q$-integrals of (4.9) tends to the $q$-integrals of (4.7) because of $\mathcal{V}(\bar{t}, \bar{x}, \bar{v}) \rightarrow \mathcal{V}(t, x, v)$ and $\int_{0}^{t_{m}}=\int_{t_{m+1}}^{t_{m}}$. On the other hand, since

$$
\bar{x}_{m+1}-\bar{t}_{m+1} \bar{v}_{m+1} \rightarrow x_{m+1}, \quad \bar{v}_{m+1} \rightarrow v_{m+1}=R\left(x_{m}\right) v_{m}
$$

we have

$$
h_{0}\left(\bar{x}_{m+1}-\bar{t}_{m+1} \bar{v}_{m+1}, \bar{v}_{m+1}\right) \rightarrow h_{0}\left(x_{m+1}, R\left(x_{m}\right) v_{m}\right)=h_{0}\left(x_{m}, v_{m}\right)
$$

from $h_{0}(x, v)=h_{0}(x, R(x) v)$ on $\gamma$. We thus complete the proof.

4.1. $\operatorname{det}\left\{\frac{\partial v_{k}}{\partial v_{\ell}}\right\}$ near $\partial \Omega$. We now compute $\operatorname{det}\left\{\frac{\partial v_{k}}{\partial v_{\ell}}\right\}$ for carefully chosen specular back-time cycle near the boundary $\partial \Omega$. Given small $\varepsilon_{0}$, we choose $v_{1}$ such that

$$
\left|v_{1}\right|=\varepsilon_{0}, \quad v_{1} \cdot n\left(x_{1}\right)=\frac{v \cdot \nabla \xi\left(x_{1}\right)}{\left|\nabla \xi\left(x_{1}\right)\right|}=\varepsilon_{0}^{2}
$$

We shall analyze the specular back-time cycle of $\left(0, x_{1}, v_{1}\right):\left(t_{k}, x_{k}, v_{k}\right)$. Lettting

$$
\xi\left(x_{1}-\sum_{j=1}^{k} s_{j} v_{j}\right)=0, \quad v_{k}=R\left(x_{k}\right) v_{k-1}, \quad x_{k}=x_{k-1}-s_{k} v_{k} \in \partial \Omega
$$

Proposition 4.5. [12] For any finite $k \geq 1$,

$$
\frac{\partial v_{k}^{i}}{\partial v_{1}^{\ell}}=\delta_{i j}+\zeta(k) n^{i}\left(x_{1}\right) n^{\ell}\left(x_{1}\right)+O\left(\varepsilon_{0}\right) .
$$

where $O$ depends on $k$ and $\zeta$ is defined as $\zeta(1)=0$.

$$
\zeta(k)=4 \sum_{p=1}^{k-2}(-1)^{k-p+1}+4 \sum_{p=1}^{k-2}(-1)^{k-p-1} \zeta(p)+2+3 \zeta(k-1), \text { for } k \geq 2 .
$$

In particular, $\zeta(t)$ is an even integer so that

$$
\operatorname{det}\left(\frac{\partial v_{k}^{i}}{\partial v_{1}^{\ell}}\right)=\{\zeta(k)+1\}+O\left(\varepsilon_{0}\right) \neq 0
$$


4.2. $L^{\infty}$ Decay for $U(t)$. From Duhamel's principle, we have

$$
\begin{aligned}
h(t)= & G(t) h_{0}+\int_{0}^{t} G(t-s) K_{w_{\varpi}}^{x, \varpi} h(s) d s_{1} \\
= & G(t) h_{0}+\int_{0}^{t} G\left(t-s_{1}\right) K_{w_{\varpi}}^{x, \varpi} G\left(s_{1}\right) h\left(s_{1}\right) d s_{1} \\
& +\int_{0}^{t} \int_{0}^{s_{1}} G\left(t-s_{1}\right) K_{w_{\varpi}}^{x, \varpi} G\left(s_{1}-s\right) K_{w_{\varpi}}^{x, \varpi} h(s) d s d s_{1}
\end{aligned}
$$

Therefore, $h$ can be written as follows

$$
\begin{aligned}
h(t, x, v)= & e^{-\mathcal{V}(t, x, v)} h_{0}\left(X_{\mathbf{c l}}(0), V_{\mathbf{c l}}(0)\right) \\
+ & \int_{0}^{t} e^{-\mathcal{V}\left(t-s_{1}, x, v\right)} \int K_{w}^{\varpi, X_{\mathbf{c l}}\left(s_{1}\right)}\left(V_{\mathbf{c l}}\left(s_{1}\right), v^{\prime}\right) e^{-\mathcal{V}\left(s_{1}, X_{\mathbf{c l}}\left(s_{1}\right), v^{\prime}\right.} h_{0}\left(X_{\mathbf{c l}}^{\prime}(0), V_{\mathbf{c l}}^{\prime}(0)\right) d v^{\prime} \\
+ & \int_{0}^{t} \int_{0}^{s_{1}} \int_{R^{6}} e^{-\mathcal{V}\left(t-s_{1}, x, v\right)-\mathcal{V}\left(s_{1}-s, X_{\mathbf{c l}}\left(s_{1}\right), v^{\prime}\right)} \\
& \quad \times K_{w_{\varpi}, X_{\mathbf{c l}}\left(s_{1}\right)}^{V_{1}}\left(V_{\mathbf{c l}}\left(s_{1}\right), v^{\prime}\right) K_{w_{\varpi}}^{\varpi, X_{\mathbf{c l}}^{\prime}(s)}\left(V_{\mathbf{c l}}^{\prime}(s), v^{\prime \prime}\right) h\left(s, X_{\mathbf{c l}}^{\prime}(s), v^{\prime \prime}\right) d s d s_{1} d v^{\prime} d v^{\prime \prime} . \\
\equiv & (\mathcal{A})_{\varpi}+\mathcal{B}_{\varpi}+\mathcal{C}_{\varpi} .
\end{aligned}
$$

where the back-time specular cycle from $\left(s_{1}, X_{\mathbf{c l}}\left(s_{1}\right), v^{\prime}\right)$ is denoted by

$$
X_{\mathbf{c l}}^{\prime}(s)=X_{\mathbf{c l}}\left(s ; s_{1}, X_{\mathbf{c l}}\left(s_{1}\right), v^{\prime}\right), \quad V_{\mathbf{c l}}^{\prime}=V_{\mathbf{c l}}\left(s ; s_{1}, X_{\mathbf{c l}}\left(s_{1}\right), v^{\prime}\right) .
$$

More explicitly, let $t_{k}$ and $t_{k}^{\prime}$ be the corresponding times for both specular cycles as in (4.2).

For $t_{k+1} \leq s \leq t_{k}, t_{k^{\prime}+1}^{\prime} \leq s \leq t_{k^{\prime}}^{\prime}$

$$
X_{\mathbf{c l}}^{\prime}(s)=X_{\mathbf{x l}}\left(s ; s_{1}, X_{\mathbf{c l}}\left(s_{1}\right), v^{\prime}\right) \equiv x_{k^{\prime}}+\left(s-t_{k^{\prime}}^{\prime}\right) v_{k^{\prime}}^{\prime},
$$

where

$$
x_{k^{\prime}}^{\prime}=X_{\mathbf{c l}}\left(t_{k^{\prime}}: s_{1}, x_{k}+\left(s_{1}-t_{k}\right) v_{k}, v^{\prime}\right), \quad v_{k^{\prime}}^{\prime}=V_{\mathbf{c l}}\left(t_{k^{\prime}}: s_{1}, x_{k}+\left(s_{1}-t_{k}\right) v_{k}, v^{\prime}\right) .
$$

The collision frequency $\mathcal{V}$ is defined as in the proof of Lemma 4.4;

$$
\begin{aligned}
\mathcal{V}(t, x, v)= & \nu^{\varpi}(x, v)\left(t-t_{1}\right)+\nu^{\varpi}\left(x_{1}, v_{1}\right)\left(t_{1}-t_{2}\right)+ \\
& \cdots+\nu^{\varpi}\left(x_{m-1}, v_{m-1}\right)\left(t_{m-1}-t_{m}\right)+\nu^{\varpi}\left(x_{m}, v_{m}\right) t_{m} .
\end{aligned}
$$

Recall

$$
\alpha(s)=\xi^{2}(X(s))+[V(x) \cdot \nabla \xi(X(s))]^{2}-2\{V(s) \cdot \xi(X(s)) \cdot V(s)\} \xi(X(x)) .
$$

We are now in a place to state the main theorem of this section.

Theorem 4.6. Assume $w_{\varpi}^{-2}(1+|v-\varpi \times x|) \in L^{1}$. Assume that $\Omega$ satisfies the geometric assumption $(\mathcal{A})$ and the mass, angular momentum and energy are conserved as in (1.10). Then there exists a unique solution $f(t, x, v)$ to

$$
\begin{array}{r}
\left\{\partial_{t}+v \cdot \nabla_{x}+\nu^{\varpi}-K^{\varpi, x}\right\} f=0, \quad f(0, v, x)=f_{0}, \\
\left.f(t, x, v)\right|_{\gamma_{+}}=\left.f(t, x, R(x) v)\right|_{\gamma_{-}} .
\end{array}
$$

and $h(t, x, v)=U(t) h_{0}$ to the weighted linear Boltzmann equation:

$$
\begin{gathered}
\left\{\partial_{t}+v \cdot \nabla_{x}+\nu^{\varpi}-K_{w_{\varpi}}^{\varpi, x}\right\}\left\{U(t, 0) h_{0}\right\}=0, \quad U(0,0) h_{0}=h_{0}, \\
\left.\left\{U(t) h_{0}\right\}(t, x, v)\right|_{\gamma_{+}}=\left.\left\{U(t) h_{0}\right\}(t, x, R(x) v)\right|_{\gamma_{-}} .
\end{gathered}
$$

Moreover, there exists $0<\lambda<\lambda_{0}$ such that

$$
\sup _{t \geq 0} e^{\lambda t}\left\|U(t, 0) h_{0}\right\|_{\infty} \leq C\left\|h_{0}\right\|_{\infty}
$$


For the proof of the theorem, we need the following two technical lemmas. The proof can be found in 12 .

Lemma 4.7. Assume that there exists $\lambda>0$ so that the solution $f(t, x, v)$ of the linearized Boltzmann equation (3.1) satisfies $e^{\lambda t}\|f(t)\| \leq C\left\|f_{0}\right\|$. Let $h_{0}=w f_{0} \in L^{\infty}$ and $h(t)=$ $U(t) h_{0}=w f(t)$ is the solution of (4.12) where $w^{-2} \in L^{1}$. Assume there exist $T_{0}>0$ and $C_{T_{0}}>0$ such that

$$
\left\|U\left(T_{0}\right) h_{0}\right\|_{\infty} \leq e^{-\lambda T_{0}}\left\|h_{0}\right\|_{\infty}+C_{T_{0}} \int_{0}^{T_{0}}\|f(s)\| d s .
$$

Then we have for all $t \geq 0$

$$
\sup _{t \geq 0} e^{\lambda t}\left\|U(t, 0) h_{0}\right\|_{\infty} \leq C\left\|h_{0}\right\|_{\infty} .
$$

Proof. See [12.

We now define the main set:

$$
A_{\alpha}(x, v)=\left\{(x, v): x \in \bar{\Omega}, \frac{1}{2 N} \leq|v| \leq 2 N, \text { and } \alpha(x, v) \geq \frac{1}{N}\right\} .
$$

Lemma 4.8. Fix $k$ and $k^{\prime}$. Define for $t_{k+1} \leq s_{1} \leq t_{k}$ and $s \in R$

$$
J=J_{k, k^{\prime}}\left(t, x, v, s_{1}, s, v^{\prime}\right)=\operatorname{det}\left(\frac{\partial\left\{x_{k^{\prime}}^{\prime}+\left(s-t_{k^{\prime}}^{\prime}\right) v_{k^{\prime}}^{\prime}\right\}}{\partial v^{\prime}}\right) .
$$

For any $\varepsilon>0$ sufficiently small, there is $\delta\left(N, \varepsilon, T_{0}, k, k^{\prime}\right)>0$ and an open covering $\cup_{i=1}^{m} B\left(t_{i}, x_{i}, v_{i}\right.$ : $\left.r_{i}\right)$ of $\left[0, T_{0}\right] \times A_{\alpha}$ and corresponding open sets (with $\left.x_{i} \in \bar{\Omega}\right) O_{t_{i}, x_{i}, v_{i}}$ for $\left[t_{k+1}+\varepsilon, t_{k}-\varepsilon\right] \times$ $R \times \mathbb{R}^{3}$ with $\left|O_{t_{i}, x_{i}, v_{i}}\right|<\varepsilon$, such that

$$
\left|J_{k, k^{\prime}}\left(t, x, v, s_{1}, s, v^{\prime}\right)\right| \geq \delta>0,
$$

for $0 \leq t \leq T_{0},(x, v) \in A_{\alpha}$ and $\left(s_{1}, s, v^{\prime}\right)$ in

$$
O_{t_{i}, x_{i}, v_{i}}^{c} \cap\left[t_{k+1}+\varepsilon, t_{k}-\varepsilon\right] \times\left[0, T_{0}\right] \times\left\{\left|v^{\prime}\right| \leq 2 N\right\} .
$$

Proof. See [12.

Proof of Theorem 4.6: We first notice that there exists a constant $\nu_{0}$ such that $\nu_{0} \leq$ $\nu\left(x_{i}, v_{i}\right)$ and

$$
\begin{aligned}
-\mathcal{V}(t, x, v) & \leq-\nu_{0}\left(t-t_{1}\right)-\nu_{0}\left(t_{1}-t_{2}\right)-\cdots-\nu_{0}\left(t_{m-1}-t_{m}\right)-\nu_{0} t_{m} \\
& =-\nu_{0} t .
\end{aligned}
$$

From this, the estimates for $(\mathcal{A})_{\varpi}$ :

$$
(\mathcal{A})_{\varpi} \leq e^{-\nu_{0} t}\left\|h_{0}\right\|_{\infty}
$$

For the second term $\mathcal{B}_{\varpi}$ in (4.10), we note that $\left\|K_{w}^{\varpi, x} h\right\|_{\infty} \leq C\|h\|_{\infty}$. Then by Lemma 2.3,

$$
\begin{aligned}
\mathcal{B}_{\varpi} & =\left\|\int_{0}^{t} G\left(t-s_{1}\right) K_{\varpi}^{\varpi, x} G\left(s_{1}\right) h_{0} d s_{1}\right\|_{\infty} \\
& \leq \int_{0}^{t} e^{-\nu_{0}\left(t-s_{1}\right)}\left\|K_{\varpi}^{\varpi, x} G\left(s_{1}\right) h_{0}\right\|_{\infty} d s_{1} \\
& \leq C t e^{-\nu_{0} t}\left\|h_{0}\right\|_{\infty} .
\end{aligned}
$$

We now concentrate on $\mathcal{C}_{\varpi} \mathbf{1}_{A_{\alpha}}$. For this, we divide $A_{\alpha}$ into the following two cases. We suppose $N$ is large enough such that

$$
N>|\varpi| \max _{x \in \Omega}|x|
$$


Case 1: For $\{|v-\varpi \times x| \leq 3 N\} \cap\left\{\left|v^{\prime}-\varpi \times x\right| \geq 6 N\right\} \cap A_{\alpha}$ or $\left\{\left|v^{\prime}-\varpi \times x\right| \leq 6 N\right\} \cap\left\{\mid v^{\prime \prime}-\right.$ $\varpi \times x \mid \geq 9 N\} \cap A_{\alpha}$.

For the first case, we recall $|v|=\left|V_{\mathbf{c l}}\left(s_{1}\right)\right|$ to see

$$
\begin{aligned}
\left|V_{\mathbf{c l}}\left(s_{1}\right)-v^{\prime}\right| & \geq\left|v^{\prime}\right|-\left|V_{\mathbf{c l}}\left(s_{1}\right)\right| \\
& =\left|v^{\prime}\right|-|v| \\
& \geq\left|v^{\prime}-\varpi \times x\right|-|v-\varpi \times x|-2|\varpi| \max _{x \in \Omega}|x| \\
& \geq 6 N-3 N-2 N=N .
\end{aligned}
$$

In a similar way, we have for the second case

$$
\left|V_{\mathbf{c l}}^{\prime}(s)-v^{\prime \prime}\right| \geq N .
$$

Therefore either one of the following are valid correspondingly:

$$
\begin{gathered}
\left|K_{w_{\varpi}}^{\varpi, X_{\mathbf{c l}}\left(s_{1}\right)}\left(V_{\mathbf{c l}}\left(s_{1}\right), v^{\prime}\right)\right| \leq e^{-\frac{\varepsilon}{8} N^{2}}\left|K_{w_{\varpi}}^{\varpi, X_{\mathbf{c l}}\left(s_{1}\right)}\left(V_{\mathbf{c l}}\left(s_{1}\right), v^{\prime}\right)\right| e^{\frac{\varepsilon}{8}\left|V_{\mathbf{c l}}\left(s_{1}\right)-v^{\prime}\right|^{2}}, \\
\left|K_{w_{\varpi}}^{\varpi, X_{\mathbf{c l}}^{\prime}(s)}\left(V_{\mathbf{c l}}^{\prime}(s), v^{\prime \prime}\right)\right| \leq e^{-\frac{\varepsilon}{8} N^{2}}\left|K_{w_{\varpi}}^{\varpi, X_{\mathbf{c l}}^{\prime}(s)}\left(V_{\mathbf{c l}}^{\prime}(s), v^{\prime \prime}\right)\right| e^{\frac{\varepsilon}{8}\left|V_{\mathbf{c l}}^{\prime}(s)-v^{\prime \prime}\right|^{2}} .
\end{gathered}
$$

From Lemma 2.3

$$
\begin{aligned}
& \int\left|K_{w_{\varpi}}^{\varpi \times X_{\mathbf{c l}}\left(s_{1}\right)}\left(V_{\mathbf{c l}}\left(s_{1}\right), v^{\prime}\right)\right| e^{\frac{\varepsilon}{8}\left|V_{\mathbf{c l}}\left(s_{1}\right)-v^{\prime}\right|^{2}} d v^{\prime}<\infty, \\
& \int\left|K_{w_{\varpi}}^{\varpi, X_{\mathbf{c l}}^{\prime}(s)}\left(V_{\mathbf{c l}}^{\prime}(s), v^{\prime \prime}\right)\right| e^{\frac{\varepsilon}{8}\left|V_{\mathbf{c l}}^{\prime}(s)-v^{\prime \prime}\right|^{2}} d v^{\prime \prime}<\infty .
\end{aligned}
$$

Using this, we split the estimate as follows

$$
\begin{aligned}
\int_{0}^{t} & \int_{0}^{s_{1}}\left\{\int_{\substack{|v-\varpi \times x| \leq 3 N,\left|v^{\prime}-\varpi \times x\right| \geq 6 N}}+\int_{\substack{\left|v^{\prime}-\varpi \times x\right| \leq 6 N,\left|v^{\prime \prime}-\varpi \times x\right| \geq 9 N}}\right\} \\
\leq & C_{K} \int_{0}^{t} \int_{0}^{s_{1}} e^{-\nu_{0}(t-s)}\left\|U(s, 0) h_{0}\right\|_{\infty}\left\{\sup _{v} \int K_{w_{\varpi}}^{\varpi, X_{\mathbf{c l}}\left(s_{1}\right)}\left(V_{\mathbf{c l}}\left(s_{1}\right), v^{\prime}\right) d v^{\prime}\right. \\
& \left.\quad+\sup _{v^{\prime}} \int K_{w_{\varpi}}^{\varpi, X_{\mathbf{c l}}^{\prime}(s)}\left(V_{\mathbf{c l}}^{\prime}(s), v^{\prime \prime}\right) d v^{\prime \prime}\right\} \\
\leq & C_{\varepsilon, K} e^{-\frac{\varepsilon}{8} N^{2}} \int_{0}^{t} \int_{0}^{s_{1}} e^{-\nu_{0}(t-s)}\left\|U(s, 0) h_{0}\right\|_{\infty} d s d s_{1} \\
\leq & C_{\varepsilon, K} e^{-\frac{\varepsilon}{8} N^{2}} \sup _{s \geq 0}\left\{e^{\frac{\nu_{0}}{2} s}\left\|U(s, 0) h_{0}\right\|_{\infty}\right\} .
\end{aligned}
$$

Case 2: $\left\{\left|v^{\prime}-\varpi \times x\right| \leq 6 N\right\} \cap\left\{\left|v^{\prime \prime}-\varpi \times x\right| \leq 9 N\right\} \cap A_{\alpha}$.

We have from $\left|V_{\mathbf{c l}}\left(s_{1}\right)\right|=|v|$

$$
\begin{aligned}
\left|V_{\mathbf{c l}}\left(s_{1}\right)-\varpi \times X_{\mathbf{c l}}\left(s_{1}\right)\right| & \leq\left|V_{\mathbf{c l}}\left(s_{1}\right)\right|+|\varpi|\left|X_{\mathbf{c l}}\left(s_{1}\right)\right| \\
& \leq|v|+|\varpi| \max _{x \in \Omega}|x| \\
& \leq|v-\varpi \times x|+2|\varpi| \max _{x \in \Omega}|x| \\
& \leq 3 N+2 N=5 N .
\end{aligned}
$$

Likewise,

$$
\left|V_{\mathbf{c l}}^{\prime}(s)-\varpi \times X_{\mathbf{c l}}^{\prime}\left(s_{1}\right)\right| \leq 8 N
$$


We can choose $K_{w N}^{\varpi, x}\left(v, v^{\prime}\right)$ smooth with compact support such that

$$
\begin{aligned}
& \sup _{\substack{x \in \Omega,|p-\varpi \times x| \leq 3 N}} \int_{\left|v^{\prime}-\varpi \times x\right| \leq 5 N}\left|K_{w_{\varpi}}^{\varpi, x}\left(p, v^{\prime}\right)-K_{N}^{\varpi, x}\left(p, v^{\prime}\right)\right| d v^{\prime} \leq \frac{1}{N}, \\
& \sup _{\substack{x \in \Omega,|p-\varpi \times x| \leq 6 N}} \int_{\left|v^{\prime}-\varpi \times x\right| \leq 8 N}\left|K_{w_{\varpi}}^{\varpi, x}\left(p, v^{\prime}\right)-K_{N}^{\varpi, x}\left(p, v^{\prime}\right)\right| d v^{\prime} \leq \frac{1}{N} .
\end{aligned}
$$

We use this to obtain the following splitting:

$$
\begin{aligned}
& K_{w_{\varpi}, X_{\mathbf{c l}}\left(s_{1}\right)}\left(V_{\mathbf{c l}}\left(s_{1}\right), v^{\prime}\right) K_{w_{\varpi}}^{\varpi, X_{\mathbf{c l}}^{\prime}(s)}\left(V_{\mathbf{c l}}^{\prime}(s), v^{\prime \prime}\right) \\
& \quad=\left\{K_{w_{\varpi}}^{\varpi, X_{\mathbf{c l}}\left(s_{1}\right)}\left(V_{\mathbf{c l}}\left(s_{1}\right), v^{\prime}\right)-K_{N}^{\varpi, X_{\mathbf{c l}}\left(s_{1}\right)}\left(V_{\mathbf{c l}}\left(s_{1}\right), v^{\prime}\right)\right\} K_{w}^{\varpi, X_{\mathbf{c l}}^{\prime}(s)}\left(V_{\mathbf{c l}}^{\prime}(s), v^{\prime \prime}\right) \\
& \quad+K_{w_{\varpi}}^{\varpi, X_{\mathbf{c l}}\left(s_{1}\right)}\left(V_{\mathbf{c l}}\left(s_{1}\right), v^{\prime}\right)\left\{K_{N}^{\varpi, X_{\mathbf{c l}}^{\prime}(s)}\left(V_{\mathbf{c l}}(s), v^{\prime \prime}\right)-K_{N}^{\varpi, X_{\mathbf{c l}}^{\prime}(s)}\left(V_{\mathbf{c l}}^{\prime}(s), v^{\prime \prime}\right)\right\} \\
& \quad+K_{N}^{\varpi, X_{\mathbf{c l}}\left(s_{1}\right)}\left(V_{\mathbf{c l}}\left(s_{1}\right), v^{\prime}\right) K_{N}^{\varpi, X_{\mathbf{c l}}^{\prime}(s)}\left(V_{\mathbf{c l}}^{\prime}(s), v^{\prime \prime}\right) .
\end{aligned}
$$

We can use the approximation (4.16) to estimate as

$$
\begin{aligned}
\mathcal{C}_{\varpi} \mathbf{1}_{A_{\alpha}}= & \frac{C e^{-\frac{\nu_{0} t}{2}}}{N} \sup _{s}\left\{e^{\frac{\nu_{0} s}{2}}\left\|U(s, 0) h_{0}\right\|_{\infty}\right\} \\
& \times\left\{\sup _{v} \int_{\left|v^{\prime}-X_{\mathbf{c l}}\left(s_{1}\right)\right| \leq 5 N} K_{w_{\varpi}, X_{\mathbf{c l}}\left(s_{1}\right)}\left(V_{\mathbf{c l}}\left(s_{1}\right), v^{\prime}\right) d v^{\prime}\right. \\
& \left.\quad+\sup _{v^{\prime}} \int_{\left|v^{\prime \prime}-\varpi \times X_{\mathbf{c l}}^{\prime}(s)\right| \leq 8 N} K_{w_{\varpi}, X_{\mathbf{c l}}^{\prime}(s)}\left(V_{\mathbf{c l}}^{\prime}(s), v^{\prime \prime}\right) d v^{\prime \prime}\right\} \\
& +\int_{0}^{t} \int_{0}^{s_{1}} \int_{\left|v^{\prime}-\varpi \times x\right| \leq 6 N,} e^{-\nu_{0}(t-s)} K_{N}^{\varpi, X_{\mathbf{c l}}\left(s_{1}\right)}\left(V_{\mathbf{c l}}\left(s_{1}\right), v^{\prime}\right) K_{N}^{\varpi, X_{\mathbf{c l}}^{\prime}(s)}\left(V_{\mathbf{c l}}^{\prime}(s), v^{\prime \prime}\right) \\
& \frac{C e^{-\frac{\nu_{0} t}{2}}}{N^{\prime \prime}} \sup _{s}\left\{e^{\frac{\nu_{0} s}{2}}\left\|U(s, 0) h_{0}\right\|_{\infty}\right\} \\
& +\int_{0}^{t} \int_{0}^{s_{1}} \int_{\left|v^{\prime}-\varpi \times x\right| \leq 6 N,} e^{-\nu_{0}(t-s)} \mathbf{1}_{A_{\alpha} \mid}\left|h\left(s, X_{\mathbf{c l}}^{\prime}(s), v^{\prime \prime}\right)\right| d v^{\prime} d v^{\prime \prime} d s_{1} d s,
\end{aligned}
$$

where we used the boundedness of

$$
K_{N}^{\varpi, X_{\mathbf{c l}}\left(s_{1}\right)}\left(V_{\mathbf{c l}}\left(s_{1}\right), v^{\prime}\right) K_{N}^{\varpi, X_{\mathbf{c l}}^{\prime}(s)}\left(V_{\mathbf{c l}}^{\prime}(s), v^{\prime \prime}\right) .
$$

We further divide the estimate as

$$
\begin{aligned}
\int_{0}^{t} \int_{0}^{s_{1}} & \int_{\substack{\left|v^{\prime}-\varpi \times x\right| \leq 6 N,\left|v^{\prime \prime}-\varpi \times x\right| \leq 9 N}} e^{-\nu_{0}(t-s)} \mathbf{1}_{A_{\alpha}}\left|h\left(s, X_{\mathbf{c l}}^{\prime}(s), v^{\prime \prime}\right)\right| d v^{\prime} d v^{\prime \prime} d s_{1} d s \\
& \leq \int_{\substack{\alpha\left(X_{\mathbf{c l}}\left(s_{1}\right), v^{\prime}\right)<\varepsilon \\
\left|v^{\prime}-\varpi \times x\right| \leq 6 N,\left|v^{\prime \prime}-\varpi \times x\right| \leq 9 N}}+\int_{\substack{\alpha\left(X_{\mathbf{c l}}\left(s_{1}\right), v^{\prime}\right) \geq \varepsilon \\
\left|v^{\prime}-\varpi \times x\right| \leq 6 N,\left|v^{\prime \prime}-\varpi \times x\right| \leq 9 N}} \quad I_{1}+I_{2} .
\end{aligned}
$$


In the case $\alpha\left(X_{\mathbf{c l}}\left(s_{1}\right), v^{\prime}\right) \leq \varepsilon, \xi^{2}\left(X_{\mathbf{c l}}\left(s_{1}\right)\right)+\left[v^{\prime} \cdot \nabla \xi\left(X_{\mathbf{c l}}\left(s_{1}\right), v^{\prime}\right)\right] \leq \varepsilon$. Hence for small $\varepsilon$, $X_{\mathbf{c l}}\left(s_{1}\right) \sim \partial \Omega$ and $\left|\nabla \xi\left(X_{\mathbf{c l}}\left(s_{1}\right)\right)\right| \geq \frac{1}{2}$. The first term $I_{1}$ is bounded by

$$
\begin{aligned}
& I_{1}=C_{N} \int_{0}^{t} \int_{0}^{s_{1}} e^{-\nu_{0}(t-s)}\|h(s)\|_{\infty} d s d s_{1} \int_{\left|v^{\prime}-\varpi \times x\right| \leq 6 N,\left|v^{\prime \prime}-\varpi \times x\right| \leq 9 N}^{\alpha\left(X_{\mathrm{cl}}\left(s_{1}\right), v^{\prime}\right)<\varepsilon} \\
& \leq C_{N} \sup _{t \geq s} e^{-\frac{\nu_{0}}{2}(t-s)}\|h(s)\|_{\infty} \int_{\left|v^{\prime}-\varpi \times x\right| \leq 6 N,\left|v^{\prime \prime}-\varpi \times x\right| \leq 9 N} \underset{\left|v^{\prime} \cdot \frac{\nabla \xi\left(X_{\mathrm{cl}}\left(s_{1}\right), v^{\prime}\right)}{\left|\nabla \xi\left(X_{\mathrm{c}}\right)\left(s_{1}\right)\right|}\right| \leq \varepsilon}{ }
\end{aligned}
$$

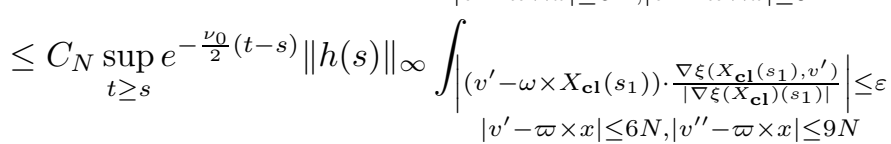

$$
\begin{aligned}
& \leq C_{N} \varepsilon \sup _{t \geq s} e^{-\frac{\nu_{0}}{2}(t-s)}\|h(s)\|_{\infty},
\end{aligned}
$$

where we used the rotational symmetry:

$$
\omega \times X_{\mathbf{c l}}\left(s_{1}\right) \cdot \frac{\nabla \xi\left(X_{\mathbf{c l}}\left(s_{1}\right), v^{\prime}\right)}{\left|\nabla \xi\left(X_{\mathbf{c l}}\right)\left(s_{1}\right)\right|}=0 .
$$

On the other hand, to estimate $I_{2}$, we first note that

$$
\begin{aligned}
& \left|v^{\prime}\right| \leq\left|v^{\prime}-\varpi \times x\right|+|\varpi| \max _{x \in \Omega}|x| \leq 7 N, \\
& \left|v^{\prime \prime}\right| \leq\left|v^{\prime \prime}-\varpi \times x\right|+|\varpi| \max _{x \in \Omega}|x| \leq 10 N .
\end{aligned}
$$

From (4.17), we can write $I_{2}$ as

$$
\begin{aligned}
I_{2} & \leq C_{N} \int_{0}^{t} \int_{0}^{s_{1}} \int_{\substack{\alpha\left(X_{\mathrm{cl}}\left(s_{1}\right), v^{\prime}\right) \geq \varepsilon \\
\left|v^{\prime}\right| \leq 7 N,\left|v^{\prime \prime}\right| \leq 10 N}} e^{-\nu_{0}(t-s)} \mathbf{1}_{A_{\alpha}}\left|h\left(s, X_{\mathbf{c l}}^{\prime}(s), v^{\prime \prime}\right)\right| d v^{\prime} d v^{\prime \prime} d s_{1} d s, \\
& \left.=C_{N} \sum_{k, k^{\prime}} \int_{t_{k+1}(v)}^{t_{k}(v)} \int_{t_{k^{\prime}+1}^{\prime}\left(v^{\prime}\right)}^{t^{\prime}\left(v^{\prime}\right)} \int_{\substack{\alpha\left(X_{\mathrm{cl}}\left(s_{1}\right), v^{\prime}\right) \geq \varepsilon \\
\left|v^{\prime}\right| \leq 7 N,\left|v^{\prime \prime}\right| \leq 10 N}} \mathbf{1}_{A_{\alpha}} e^{-\nu_{0}(t-s)} \mid h\left(s, x_{k^{\prime}}^{\prime}+\left(s-t_{k^{\prime}}^{\prime}\right)\right) v_{k^{\prime}}^{\prime}, v^{\prime \prime}\right) \mid .
\end{aligned}
$$

Therefore, in a small velocity regime, the rotation does not have big effect and we can consider this problem as in [12. We now study $x_{k^{\prime}}^{\prime}+\left(s-t_{k^{\prime}}^{\prime}\right) v_{k^{\prime}}^{\prime}$. By repeatedly using Lemma 2.1, we deduce for $(t, s, v) \in \bar{A}_{\alpha}$ and $\alpha\left(X\left(s_{1}\right), v^{\prime}\right) \geq \varepsilon,\left|v^{\prime}\right| \leq 7 N$ :

$$
\begin{aligned}
& \alpha\left(t_{\ell}\right)=\left\{v_{\ell} \cdot n_{x_{\ell}}\right\} \geq e^{-\left\{C_{\xi} N-1\right\} T_{0}} \alpha\left(s_{1}\right) \geq C_{T_{0}, \xi, N}>0 ; \\
& \alpha\left(t_{\ell}^{\prime}\right)=\left\{v_{\ell}^{\prime} \cdot n_{x_{\ell}^{\prime}}\right\} \geq e^{-\left\{C_{\xi} N-1\right\} T_{0}} \alpha\left(X_{\mathbf{c l}}\left(s_{1}\right), v^{\prime}\right) \geq C_{T_{0}, \xi, N, \varepsilon}>0 .
\end{aligned}
$$

Therefore, applying Lemma 2.2 (3) yields

$$
t_{\ell}-t_{\ell+1} \geq \frac{C_{T_{0}, \varepsilon, N}}{N^{2}}, \quad t_{\ell}^{\prime}-t_{\ell+1}^{\prime} \geq \frac{C_{T_{0}, \varepsilon, N, \varepsilon}}{4 N^{2}},
$$

So that

$$
k \leq \frac{T_{0} N^{2}}{C_{T_{0}, \varepsilon, N}}=C_{T_{0}, \varepsilon, N}, \quad k^{\prime} \leq \frac{T_{0} N^{2}}{C_{T_{0}, \varepsilon, N, \varepsilon}}=C_{T_{0}, \xi, N, \varepsilon} .
$$

We therefore further split the $s_{1}$-ingegral as

$$
\left.C_{k, N} \int_{t_{k+1}}^{t_{k}} \int_{\substack{\left|v^{\prime}\right| \leq 7 N,\left|v^{\prime \prime}\right| \leq 10 N}} \sum_{\substack{k \leq C_{T_{0}, \varepsilon, N}, k^{\prime} \leq C_{T_{0}, \varepsilon, N}}} \int_{t_{k^{\prime}+1}^{\prime}}^{t^{\prime}} \mathbf{1}_{A_{\alpha}} e^{-\nu_{0}(t-s)} \mid h\left(s, x_{k^{\prime}}^{\prime}+\left(s-t_{k^{\prime}}^{\prime}\right)\right) v_{k^{\prime}}^{\prime}, v^{\prime \prime}\right) \mid
$$




$$
=\int_{t_{k+1}+\varepsilon}^{t_{k}-\varepsilon}+\int_{t_{k}}^{t_{k}-\varepsilon}+\int_{t_{k+1}}^{t_{k+1}+\varepsilon}
$$

Since $\sum_{k^{\prime}} \int_{t_{k^{\prime}+1}^{\prime}}^{t_{\prime}^{\prime}}=\int_{0}^{s_{1}}$, the last two terms above make small contribution as

$$
\varepsilon C_{K, N} \sup _{0 \leq s \leq t} e^{-\nu_{0}(t-s)}\|h(s)\|_{\infty} \int_{0}^{T_{0}} \int_{\substack{\left|v^{\prime}\right| \leq 7 N,\left|v^{\prime \prime}\right| \leq 10 N}}=\varepsilon C_{K, N} \sup _{0 \leq s \leq t} e^{-\nu_{0}(t-s)}\|h(s)\|_{\infty} .
$$

For the main contribution $\int_{t_{k+1}+\varepsilon}^{t_{k}-\varepsilon}$, we fixed both $k$ and $k^{\prime}$. By lemma 4.8 , on the set $O_{t_{i}, x_{i}, v_{i}}^{c} \cap$ $\left[t_{k+1}+\varepsilon, t_{k}-\varepsilon\right] \times\left[0, T_{0}\right] \times\left\{\left|v^{\prime}\right| \leq N\right\}$, we can define a change of variable

$$
y_{k^{\prime}}=x_{k^{\prime}}^{\prime}+\left(s-t_{k^{\prime}}^{\prime}\right) v_{k^{\prime}}^{\prime}
$$

so that

$$
\operatorname{det}\left(\frac{\partial y_{k^{\prime}}}{\partial v^{\prime}}\right)>\delta_{N, T_{0}, \varepsilon, k, k^{\prime}}
$$

On $O_{t_{i}, x_{i}, v_{i}}^{c} \cap\left[t_{k+1}+\varepsilon, t_{k}-\varepsilon\right] \times\left[0, T_{0}\right] \times\left\{\left|v^{\prime}\right| \leq N\right\}$. By the Implicit Function Theorem, there are a finite open covering $\cup_{j=1}^{m} V_{j}$ of $O_{t_{i}, x_{i}, v_{i}}^{c} \cap\left[t_{k+1}+\varepsilon, t_{k}-\varepsilon\right] \times\left[0, T_{0}\right] \times\left\{\left|v^{\prime}\right| \leq N\right\}$, and smooth function $F_{j}$ such that $v^{\prime}=F_{j}\left(t, x, v, y, s_{1}, s\right)$ in $V_{j}$. We therefore have

$$
\begin{aligned}
& \sum_{\substack{k \leq C_{T_{0}, \varepsilon, N}, k^{\prime} \leq C_{T_{0}, \varepsilon, N}}} \int_{t_{k+1}}^{t} \int_{\left|v^{\prime}\right| \leq 3 N} \int_{t_{k^{\prime}+1}^{\prime}}^{t^{\prime}} \\
& \quad \leq \sum_{\substack{k \leq C_{T_{0}, \varepsilon, N} \\
k^{\prime} \leq C_{T_{0}, \varepsilon, N}}}^{t} \int_{t_{k+1}}^{t} \int_{\left|v^{\prime}\right| \leq 3 N} \int_{t_{k^{\prime}+1}^{\prime}}^{t^{\prime}} \mathbf{1}_{O_{t_{i}, x_{i}, v_{i}}}+\sum_{\substack{j, k \leq C_{T_{0}, \varepsilon, N}, k^{\prime} \leq C_{T_{0}, \varepsilon, N}}} \int_{t_{k+1}}^{t} \int_{\left|v^{\prime}\right| \leq 3 N} \int_{t_{k^{\prime}+1}^{\prime}}^{t^{\prime}} \mathbf{1}_{V_{j}} \\
& \quad I+I I .
\end{aligned}
$$

Since $\sum_{k^{\prime}} \int_{t_{k^{\prime}+1}^{\prime}}^{t_{k^{\prime}}^{\prime}}=\int_{0}^{s_{1}} \leq \int_{0}^{T_{0}}$ and $\left|O_{t_{i}, x_{i}, v_{i}}\right|<\varepsilon$, we have from Lemma 4.8

$$
I \leq C_{N^{\varepsilon}} e^{-\frac{\nu_{0}}{2} t} \sup _{s}\left\{e^{\frac{\nu_{0}}{2} s}\|h(s)\|_{\infty}\right\} .
$$

To estimate $I I$, we change variable $v^{\prime} \rightarrow y_{k^{\prime}}=x_{k^{\prime}}^{\prime}+\left(s-t_{k^{\prime}}^{\prime}\right) v_{k^{\prime}}^{\prime}$ on each $V_{j}$ to get

$$
\begin{aligned}
& \left.C_{\varepsilon, N} \int_{V_{j}} \int_{\left|v^{\prime \prime}\right| \leq 4 N} \int_{t_{k^{\prime}+1}^{\prime}}^{t_{k}^{\prime}\left(v^{\prime}\right)} e^{-\nu_{0}(t-s)} \mid h\left(s, x_{k^{\prime}}^{\prime}+\left(s-t_{k^{\prime}}^{\prime}\right)\right) v_{k^{\prime}}^{\prime}, v^{\prime \prime}\right) \mid d s d v^{\prime} d v^{\prime \prime} \\
& \left.\leq C_{\varepsilon, T_{0}, N} \int_{V_{j}} \int_{\left|v_{1}^{\prime \prime}\right| \leq 4 N} \int_{0}^{s_{1}} \mathbf{1}_{x_{k^{\prime}}^{\prime}+\left(s-t_{k^{\prime}}^{\prime}\right) v_{k^{\prime}}^{\prime} \in \Omega} e^{-\nu_{0}(t-s)} \mid h\left(s, x_{k^{\prime}}^{\prime}+\left(s-t_{k^{\prime}}^{\prime}\right)\right) v_{k^{\prime}}^{\prime}, v^{\prime \prime}\right) \mid d s d s_{1} d v^{\prime} d v^{\prime \prime} \\
& \leq C_{\varepsilon, T_{0}, N} \int_{0}^{s_{1}} \int_{V_{j}} \int_{\left|v^{\prime \prime}\right| \leq 4 N} \mathbf{1}_{y \in \Omega} e^{-\nu_{0}(t-s)}\left|h\left(s, y_{k^{\prime}}, v^{\prime \prime}\right)\right| \frac{1}{\left|\operatorname{det}\left\{\frac{\partial y_{k^{\prime}}}{\partial v^{\prime}}\right\}\right|} d y_{k^{\prime}} d v^{\prime \prime} d s d s_{1} \\
& \leq \frac{C_{\varepsilon, T_{0}, N}}{\delta} \int_{0}^{t} \int_{0}^{s_{1}} e^{-\nu_{0} t} \int_{\left|v^{\prime \prime}\right| \leq 4 N} \mathbf{1}_{y \in \Omega} e^{\nu_{0} s}\left\{\int_{\Omega} h^{2}\left(s, y, v^{\prime \prime}\right) \mid d y\right\}^{\frac{1}{2}} d v^{\prime \prime} d s d s_{1} \\
& \leq C_{\varepsilon, T_{0}, N, k, k^{\prime}} \int_{0}^{t}\|f(s)\| d s,
\end{aligned}
$$


where $f=\frac{h}{w_{\varpi}}$. We therefore conclude, by summing over $j, k$ and $k^{\prime}$, and collecting terms

$$
\begin{aligned}
& \left\|h(t, x, v) \mathbf{1}_{A_{\alpha}}\right\|_{\infty} \leq\left\{1+C_{K} t\right\} e^{-\nu_{0} t}\left\|h_{0}\right\|_{\infty} \\
& \quad+\left\{\frac{C}{N}+C_{N, T_{0}^{\varepsilon}}\right\} \sup _{s} e^{\frac{\nu_{0}}{2}(t-s)}\|h(s)\|_{\infty}+C_{\varepsilon, N, T_{0}} \int_{0}^{t}\|f(s)\| d s .
\end{aligned}
$$

Estimate of $h(t, s, v)$ : We now further plug (4.18) back in $h(t, x, v)=G(t, s) h_{0}+\int_{0}^{t} G(t-$ $\left.s_{1}\right) K_{w_{\varpi}}^{\varpi, x} h\left(s_{1}\right) d s_{1}$ to get

$$
\|h(t)\|_{\infty} \leq e^{-\nu_{0} t}\left\|h_{0}\right\|_{\infty}+\int_{0}^{t} e^{-\nu_{0}\{t-s\}}\left\|K_{w}^{\varpi, x} h\right\|_{\infty}\left(s_{1}\right) d s .
$$

But $\left\{K_{w}^{\varpi, x}\left(v, v^{\prime}\right) h\right\}\left(s_{1}, x, v\right)=\int K_{w}^{\varpi, x}\left(v, v^{\prime}\right)\left(v, v^{\prime}\right) h\left(v, v^{\prime}\right) d v^{\prime}$ and we split it as

$$
\int K_{w}^{\varpi, x}\left(v, v^{\prime}\right) h\left(s_{1}, x, v^{\prime}\right)\left\{1-\mathbf{1}_{A_{\alpha}\left(x, v^{\prime}\right)}\right\} d v^{\prime}+\int K_{w}^{\varpi, x}\left(v, v^{\prime}\right) h\left(s_{1}, x, v^{\prime}\right) \mathbf{1}_{A_{\alpha}\left(x, v^{\prime}\right)} d v^{\prime} .
$$

The first term in bounded by

$$
\left(\int_{\substack{\left|v^{\prime}-\varpi \times x\right| \geq N \\ \text { or }\left|v^{\prime}\right| \leq \frac{1}{N}}}\left|K_{w_{\varpi}}^{\varpi, x}\left(v, v^{\prime}\right)\right| d v^{\prime}+\int_{\alpha\left(x, v^{\prime}\right) \leq \frac{1}{N}}\left|K_{w_{\varpi}}^{\varpi, x}\left(v, v^{\prime}\right)\right| d v^{\prime}\right)\left\|h\left(s_{1}\right)\right\|_{\infty} .
$$

The first term is obviously $o(1)$.From $\alpha\left(x, v^{\prime}\right) \leq \frac{1}{N}$, we have $\xi^{2}(x)+\left[v^{\prime} \cdot \nabla \xi(x)\right]^{2} \leq \frac{1}{N}$. Therefore, for $N$ large, we have $x \sim \partial \Omega$. On the other hand, we have $|\nabla \xi(x)| \geq \frac{1}{2}$ and the rotational symmetry assumption implies $\varpi \times x \cdot \frac{\nabla n(x)}{|\nabla n(x)|}=0$. Therefore we have

$$
\begin{aligned}
\int_{\alpha\left(x, v^{\prime}\right) \leq \frac{1}{N}}\left|K_{w}^{\varpi, x}\left(v, v^{\prime}\right)\right| d v^{\prime} & \leq \int_{\left|v^{\prime} \cdot \frac{\nabla \xi(x)}{|\nabla \xi(x)|}\right| \leq \frac{2}{\sqrt{N}}}\left|K_{w}^{\varpi, x}\left(v, v^{\prime}\right)\right| d v^{\prime} \\
& =\int_{\left|\left(v^{\prime}-\varpi \times x\right) \cdot \frac{\nabla \xi(x)}{|\nabla \xi(x)|}\right| \leq \frac{2}{\sqrt{N}}}\left|K_{w}^{\varpi, x}\left(v, v^{\prime}\right)\right| d v^{\prime} \\
& =o(1)
\end{aligned}
$$

as $N \rightarrow \infty$. We apply (4.18) to the second term to bound $\left\|K_{w}^{\varpi, x}\left(s_{1}\right)\right\|_{\infty}$ as

$$
\left\{1+C_{K} t\right\} e^{-\nu_{0} t}\left\|h_{0}\right\|_{\infty}+\left\{o(1)+\frac{C}{N}+C_{N, T_{0}^{\varepsilon}}\right\} \sup _{s} e^{\frac{\nu_{0}}{2}(t-s)}\|h(s)\|_{\infty}+C_{\varepsilon, N, T_{0}} \int_{0}^{t}\|f(s)\| d s .
$$

Hence, by (4.19), $\|h(t)\|_{\infty}$ is bounded by

$$
\begin{aligned}
& e^{-\nu_{0} t}\left\|h_{0}\right\|_{\infty}+\int_{0}^{t} e^{-\nu_{0}}\left\{1+C_{K} s_{1}\right\}\left\|h_{0}\right\|_{\infty} \\
& \quad+\int_{0}^{t}\left\{\left\{o(1)+\frac{C}{N}+C_{N, T_{0}, \varepsilon}\right\} \sup _{s} e^{\frac{\nu_{0}}{2}(t-s)}\|h(s)\|_{\infty}+C_{\varepsilon, N, T_{0}} \int_{0}^{t}\|f(s)\| d s\right\} d s_{1} \\
& \quad \leq\left\{1+C_{K} t^{2}\right\} e^{-\nu_{0} t}\left\|h_{0}\right\|_{\infty}+C\left\{o(1)+\frac{C}{N}+C_{N, T_{0}, \varepsilon}\right\} \sup _{s \leq t}\left\{e^{\frac{\nu_{0}}{2}(t-s)}\|h(s)\|_{\infty}\right\} \\
& \quad+C_{\varepsilon, N, T_{0}} \int_{0}^{t}\|f(s)\| d s .
\end{aligned}
$$

We choose $T_{0}$ large such that $2\left\{1+C_{K} T_{0}^{2}\right\} e^{-\frac{\nu_{0}}{4}} T_{0}=e^{-\lambda T_{0}}$, for some $\lambda>0$. We then further choose $N$ large, and then $\varepsilon$ sufficiently small such that $\left\{o(1)+\frac{C}{N}+C_{N, T_{0}, \varepsilon}\right\}<\frac{1}{2}$. We then 
have

$$
\sup _{0 \leq s \leq t}\left\{e^{\frac{\nu_{0}}{4} s}\|h(s)\|_{\infty}\right\} \leq 2\left\{1+C_{K} t^{2}\right\}\left\|h_{0}\right\|_{\infty}+C_{T_{0}} \int_{0}^{t}\|f(s)\| d s .
$$

Choosing $s=t=T_{0}$, we deduce the finite-time estimate (4.13), and our theorem follows from Lemma 4.7 .

\section{NONLINEAR EXPONENTIAL DECAY}

We consider the following iteration:

$$
\begin{aligned}
\left\{\partial_{t}+v\right. & \left.\cdot \nabla_{x}+\nu^{\varpi}-K_{w_{\varpi}}^{\varpi, x}\right\} h^{m+1}=w_{\varpi} \Gamma\left(\frac{h^{m}}{w_{\varpi}}, \frac{h^{m}}{w_{\varpi}}\right), \\
& \left.h^{m+1}(t, v, x)\right|_{\gamma_{-}}=\left.h^{m+1}(t, v, R(x) v)\right|_{\gamma_{+}}, \\
& h^{m+1}(0, x, v)=h_{0}(x, v), \\
& h^{0}(x, v) \equiv 0 .
\end{aligned}
$$

By the Duhamel Principle

$$
h^{m+1}=U(t) h_{0}+\int_{0}^{t} U(t-s) w_{\varpi} \Gamma\left(\frac{h^{m}}{w_{\varpi}}, \frac{h^{m}}{w_{\varpi}}\right)(s) d s .
$$

We take $L^{\infty}$ norm on both sides and apply Theorem 4.6 to obtain

$$
\left\|h^{m+1}\right\|_{\infty} \leq C e^{-\lambda t}\left\|h_{0}\right\|_{\infty}+\left\|\int_{0}^{t} U(t-s) w \Gamma\left(\frac{h^{m}}{w}, \frac{h^{m}}{w}\right)(s) d s\right\|_{\infty} .
$$

We observe

$$
U(t-s)=G(t-s)+\int_{s}^{t} G\left(t-s_{1}\right) K_{w_{\varpi}}^{\varpi, x} U\left(s_{1}-s\right) d s_{1}
$$

to get

$$
\begin{aligned}
\int_{0}^{t} U(t & -s) w_{\varpi} \Gamma\left(\frac{h^{m}}{w_{\varpi}}, \frac{h^{m}}{w_{\varpi}}\right)(s) d s \\
= & \int_{0}^{t} G(t-s) w_{\varpi} \Gamma\left(\frac{h^{m}}{w_{\varpi}}, \frac{h^{m}}{w_{\varpi}}\right)(s) d s \\
& +\int_{0}^{t} \int_{s}^{t} G(t-s) K_{w_{\varpi}, x} U\left(s_{1}-s\right) w_{\varpi} \Gamma\left(\frac{h^{m}}{w_{\varpi}}, \frac{h^{m}}{w_{\varpi}}\right) d s_{1} d s \\
& =I_{1}+I_{2} .
\end{aligned}
$$

We can estimate $I_{1}$ using Lemma 2.5 as follows

$$
\begin{aligned}
\left|\int_{0}^{t} G(t-s) w_{\varpi} \Gamma\left(\frac{h^{m}}{w_{\varpi}}, \frac{h^{m}}{w_{\varpi}}\right)(s) d s\right| & =\left|\int_{0}^{t} e^{-\mathcal{V}(t-s, x, v)}\left\{w \Gamma\left(\frac{h^{m}}{w_{\varpi}}, \frac{h^{m}}{w_{\varpi}}\right)\right\}(s, X(s), V(s)) d s\right| \\
& \leq C \int_{0}^{t} e^{-\nu_{0}(t-s)} \nu(v)\left\|h^{m}(s)\right\|_{\infty}^{2} d s \\
& \leq C e^{-\frac{\nu_{0}}{2} t}\left\{\sup _{0 \leq s \leq \infty} e^{\frac{\nu_{0}}{2} s}\left\|h^{m}(s)\right\|_{\infty}\right\}^{2} .
\end{aligned}
$$

On the other hand, for any given initial datum $\tilde{h}_{0}$, We define the semigroup $\tilde{U}(t) \tilde{h}_{0}$ as the solution operator solving the following initial boundary value problem:

$$
\left\{\partial_{t}+v \cdot \nabla_{x}+\nu^{\varpi}-K_{w_{\varpi} / \sqrt{1+|v-\varpi \times x|^{2}}}^{\varpi, x}\left\{\tilde{U}(t) \tilde{h}_{0}\right\}=0,\right.
$$




$$
\begin{aligned}
& \tilde{U}(t) \tilde{h}_{0}(t, x, v)=\tilde{U}(t) \tilde{h}_{0}(t, x, R(x) v), \\
& \tilde{U}(t) \tilde{h}_{0}=\tilde{h}_{0},
\end{aligned}
$$

where $\tilde{h}_{0}=\frac{h_{0}}{\sqrt{1+|v-\varpi \times x|^{2}}}$. Direct computation shows that $\sqrt{1+|v-\varpi \times x|^{2}} \tilde{U}(t) \tilde{h}_{0}$ solves the original linear Boltzmann equation (3.1). Therefore, we deduce from the uniqueness in the $L^{\infty}$ class:

$$
U(t) h_{0} \equiv \sqrt{1+|v-\varpi \times x|^{2}} \tilde{U}(t)\left\{\frac{h_{0}}{\sqrt{1+|v-\varpi \times x|^{2}}}\right\} .
$$

Therefore, we can rewrite $K_{w_{\varpi}}^{\varpi, x} U\left(s_{1}, s\right) w \Gamma\left(\frac{h^{m}}{w}, \frac{h^{m}}{w}\right)(s)$ to get

$$
\begin{aligned}
& \int_{0}^{t} \int_{s}^{t} G(t-s) K_{w_{\varpi}}^{\varpi, x} U\left(s_{1}-s\right) w_{\varpi} \Gamma\left(\frac{h^{m}}{w_{\varpi}}, \frac{h^{m}}{w_{\varpi}}\right) d s_{1} d s \\
& =\int_{0}^{t} \int_{s}^{t} e^{-\mathcal{V}\left(t-s_{1}, x, v\right)}\left\{\int K_{w_{\varpi}, X_{\mathbf{c l}}\left(s_{1}\right)}\left(V_{\mathbf{c l}}\left(s_{1}\right), v^{\prime}\right)\left\{\sqrt{1+\left|v^{\prime}-\varpi \times X_{\mathbf{c l}}\left(s_{1}\right)\right|^{2}}\right\} d v^{\prime}\right\} \\
& \quad \times\left\|\tilde{U}(t)\left(s_{1}-s\right)\left\{\frac{w_{\varpi}}{\sqrt{1+\left|v^{\prime}-\varpi \times x\right|^{2}}} \Gamma\left(\frac{h^{m}}{w_{\varpi}}, \frac{h^{m}}{w_{\varpi}}\right)(s)\right\}\right\|_{\infty} d s_{1} d s .
\end{aligned}
$$

Since $w_{\varpi}^{-2}(1+|v-\varpi \times x|)^{3} \in L^{1}$, the new weight $\left\{\frac{w_{\varpi}}{\sqrt{1+|v-\varpi \times x|^{2}}}\right\}^{-2}(1+|v-\varpi \times x|) \in L^{1}$ so that Theorem 4.6 is valid for $\tilde{U}$. Since $\frac{\nu\left(v^{\prime}\right)}{\sqrt{1+\left|v^{\prime}\right|^{2}}} \leq C_{\rho}$, from the proof of Lemma 2.3,

$$
\int_{\mathbb{R}^{3}} K_{w_{\varpi}}^{\varpi, X_{\mathrm{cl}}\left(s_{1}\right)}\left(V_{\mathbf{c l}}\left(s_{1}\right), v^{\prime}\right)\left\{\sqrt{1+\left|v^{\prime}-\varpi \times X_{\mathbf{c l}}\left(s_{1}\right)\right|^{2}}\right\} d v^{\prime}<+\infty .
$$

Hence, the integral can be estimated as follows

$$
\begin{aligned}
\int_{0}^{t} \int_{s}^{t} & e^{-\mathcal{V}\left(t-s_{1}, x, v\right)}\left\|\tilde{U}(t)\left(s_{1}-s\right)\left\{\frac{w_{\varpi}}{\sqrt{1+\left|v^{\prime}-\varpi \times x\right|^{2}}} \Gamma\left(\frac{h^{m}}{w_{\varpi}}, \frac{h^{m}}{w_{\varpi}}\right)(s)\right\}\right\|_{\infty} d s_{1} d s \\
& \leq C \int_{0}^{t} \int_{s}^{t} e^{-\nu_{0}(t-s)}\left\|h^{m}(s)\right\|_{\infty}^{2} d s_{1} d s \\
& \leq C e^{-\frac{\nu_{0}}{2} t}\left\{\sup _{0 \leq s \leq \infty} e^{\frac{\nu_{0}}{2} s}\left\|h^{m}(s)\right\|\right\}^{2} .
\end{aligned}
$$

This implies that

$$
\sup _{0 \leq t \leq \infty}\left\{e^{\frac{\lambda}{2} s}\left\|h^{m}(s)\right\|_{\infty}\right\} \leq C\left\|h_{0}\right\|_{\infty}
$$

for $\left\|h_{0}\right\|_{\infty}$ sufficiently small. Moreover, substracting $h^{m+1}-h^{m}$ yields

$$
\begin{aligned}
\left\{\partial_{t}+v \cdot \nabla_{x}+\right. & \nu^{\varpi}-K_{w_{\varpi}, x}^{w}\left\{\left\{h^{m+1}-h^{m}\right\}\right. \\
& =w_{\varpi} \Gamma^{\varpi}\left(\frac{h^{m}}{w_{\varpi}}, \frac{h^{m}}{w_{\varpi}}\right)-w_{\varpi} \Gamma^{\varpi}\left(\frac{h^{m-1}}{w_{\varpi}}, \frac{h^{m-1}}{w_{\varpi}}\right) \\
& =w_{\varpi} \Gamma^{\varpi}\left(\frac{h^{m}-h^{m-1}}{w_{\varpi}}, \frac{h^{m-1}}{w_{\varpi}}\right)-w_{\varpi} \Gamma^{\varpi}\left(\frac{h^{m-1}}{w_{\varpi}}, \frac{h^{m-1}-h^{m}}{w_{\varpi}}\right) .
\end{aligned}
$$

We can bound $\left\|h^{m+1}-h^{m}\right\|_{\infty}$ as in the previous estimate to obtain:

$$
C\left\|\int_{0}^{t} U_{0}(t-s) w_{\varpi} \Gamma^{\varpi}\left(\frac{h^{m}-h^{m-1}}{w_{\varpi}}, \frac{h^{m}}{w_{\varpi}}\right)(s) d s\right\|_{\infty}
$$




$$
\begin{aligned}
& +C\left\|\int_{0}^{t} U_{0}(t-s) w_{\varpi} \Gamma^{\varpi}\left(\frac{h^{m-1}}{w_{\varpi}}, \frac{h^{m-1}-h^{m}}{w_{\varpi}}\right)(s) d s\right\|_{\infty} \\
\leq & C \sup _{s}\left\{e^{\lambda s}\left\{\left\|h^{m}(s)\right\|_{\infty}\right\}+\left\|h^{m-1}\right\|_{\infty}\right\} e^{-\lambda t} \sup _{s}\left\{e^{\lambda s}\left\{\left\|h^{m}(s)-h^{m-1}(s)\right\|_{\infty}\right\}\right\} .
\end{aligned}
$$

Hence $h^{m}$ is a Cauchy sequence and the limit $h$ is the desired unique solution.

The continuity, positivity and the uniqueness of the solution can be derived by the similar argument as in [12]. Therefore we omit it.

\section{Appendix A. Proof of Theorem 1.2 (1)}

In this appendix, we consider the derivation of the rotational local Maxwellian. This proof basically relies on the argument of 12 . Suppose the following general form of local Maxwellian

$$
\mu(x, v, t)=e^{a(x, t)+b(x, t) \cdot v+c(x, t)|v|^{2}}
$$

satisfies the Boltzmann equation (1.1). Substituting (A.1) into (1.1) and using $Q(\mu, \mu)=0$, we obtain

$$
\partial_{t} \mu+v \cdot \nabla \mu=0
$$

This leads to the following system of equations for $a, b$ and $c$ for $(t, x) \in[0, \infty) \times \Omega$ :

$$
\begin{aligned}
\partial_{x_{i}} c=0, & i=1,2,3, \\
\partial_{t} c+\partial_{x_{i}} b^{i}=0, & i=1,2,3, \\
\partial_{x_{j}} b^{i}+\partial_{x_{i}} b^{j}=0, & i \neq j, \\
\partial_{t} b^{i}+\partial_{x_{i}} a=0, & i=1,2,3, \\
\partial_{t} a=0 . &
\end{aligned}
$$

This system gives a general form of $a, b, c$ ( Lemma 6 in [12]):

$$
\mu(t, x, v)=e^{\left(\frac{c_{0}}{2}|x|^{2}-b_{0} \cdot x+a_{0}\right)+\left(-c_{0} t x-c_{1} x+\varpi \times x+b_{0} t+b_{1}\right) \cdot v+\left(\frac{c_{0} t^{2}}{2}+c_{1} t+c_{2}\right)|v|^{2}}
$$

for some constants $a_{0}, b_{0}, b_{1}, c_{0}, c_{1}, c_{2}>0$. From the specular boundary condition:

$$
\mu(t, v, x)=\mu(t, x, R(x) v),
$$

we have for all $x \in \partial \Omega$ and $t \in \mathbb{R}^{+}$

$$
\left\{-c_{0} t x-c_{1} x+\varpi \times x+b_{0} t+b_{1}\right\} \cdot n(x)=0,
$$

which implies

$$
c_{0}=c_{1}=b_{0}=0 .
$$

Hence (A.3) reduces to

$$
\left\{\varpi \times x+b_{1}\right\} \cdot n(x) \equiv 0 .
$$

We now decompose $b_{1}$ as

$$
b_{1}=\beta_{1} \frac{\varpi}{|\varpi|}+\beta_{2} \eta, \text { where }|\eta|=1 \text { and } \eta \perp \varpi .
$$

Then $\eta=\left\{\frac{\varpi}{|\varpi|} \times \eta\right\} \times \frac{\varpi}{|\varpi|}$. Therefore, we get

$$
\begin{aligned}
b_{1} & =\beta_{1} \frac{\varpi}{|\varpi|}+\beta_{2}\left\{\frac{\varpi}{|\varpi|} \times \eta\right\} \times \varpi \\
& \equiv \beta_{1} \frac{\varpi}{|\varpi|}-x_{0} \times \varpi,
\end{aligned}
$$


where $x_{0}=-\beta_{2} \frac{\varpi}{|\varpi|} \times \eta$. By plugging $b_{1}$ back into (A.4), we obtain

$$
\beta_{1} \frac{\varpi}{|\varpi|} \cdot n(x)+\varpi \times\left(x-x_{0}\right) \cdot n(x)=0 .
$$

We now choose $x^{\prime}$ such that $\xi\left(x^{\prime}\right)=\min _{\xi(x)=0} \varpi \cdot x$. Then the Lagrangi Multiplier theorem implies there exists a constant $\lambda$ such that $\varpi=\lambda n\left(x^{\prime}\right)$. Setting $x=x^{\prime}$ in (A.5), we obtain $\beta_{1}=0$. Therefore, we have from (A.5)

$$
\varpi \times\left(x-x_{0}\right) \cdot n(x)=0 .
$$

We now claim that $\varpi \times x_{0}=0$. We first note that (1.3) leads to

$$
x_{1} n_{2}-x_{2} n_{1}=0
$$

which implies

$$
\left(x_{1}, x_{2}\right) / /\left(n_{1}, n_{2}\right) .
$$

Therefore, we can choose $\hat{z}, a$ and $\bar{z}$ after translation in $z$ axis, if necessary such that

$$
n((0,0, \hat{z}))=(0,0,1), \quad n((a, 0,0))=(1,0,0), \quad n((0, b, 0))=(0,1,0) .
$$

Plugging (A.7) into (A.6), we have

$$
\begin{aligned}
& \varpi_{1} x_{02}-\varpi_{2} x_{01}=0, \\
& \varpi_{2} x_{03}-\varpi_{3} x_{02}=0, \\
& \varpi_{1} x_{03}-\varpi_{3} x_{01}=0,
\end{aligned}
$$

which is equivalent to

$$
\varpi \times x_{0}=0 .
$$

We therefore have

$$
0=\varpi \times\left(x-x_{0}\right) \cdot n(x)=\varpi \times x \cdot n(x) .
$$

Now, to derive additional information on $\omega$, we perform explicit calculation using (A.6) to see

$$
\begin{aligned}
\varpi \times x \cdot n(x) & =\varpi_{1}\left(x_{2} n_{3}-x_{3} n_{2}\right)-\varpi_{2}\left(x_{1} n_{3}-x_{3} n_{1}\right)+\varpi_{3}(\underbrace{x_{1} n_{2}-x_{2} n_{1}}_{=0}) \\
& =1 / x_{1}\left(n_{1} x_{3}-n_{3} x_{1}\right)\left(\varpi_{1} x_{2}-\varpi_{2} x_{1}\right) .
\end{aligned}
$$

Hence we have either $\varpi_{1} x_{2}-\varpi_{2} x_{1}=0$ or $n_{1} x_{3}-n_{3} x_{1}=0$.

(i) The case of $\varpi_{1} x_{2}-\varpi_{2} x_{1}=0$ : The only way this identity to be true is

$$
\varpi_{1}=\varpi_{2}=0
$$

which leads to

$$
\mu(x, v)=e^{c_{0}|v|^{2}+\left(0,0, \varpi_{3}\right) \times x \cdot v+a_{0}} .
$$

(ii) The case of $n_{1} x_{3}-n_{3} x_{1}=0$ : Together with (A.6), we have for $x_{1} \neq 0$

$$
n(x)=\frac{n_{1}}{x_{1}}\left(x_{1}, x_{2}, x_{3}\right) .
$$

Hence we have

This gives

$$
1=|n(x)|=\frac{n_{1}^{2}}{x_{1}^{2}}\left(x_{1}^{2}+x_{2}^{2}+x_{3}^{2}\right) .
$$

$$
n_{1}= \pm \frac{x_{1}}{\sqrt{x_{1}^{2}+x_{2}^{2}+x_{3}^{2}}}
$$


We then put (A.9) back to (A.8) to get

$$
n(x)= \pm \frac{\left(x_{1}, x_{2}, x_{3}\right)}{\sqrt{x_{1}^{2}+x_{2}^{2}+x_{3}^{2}}}= \pm \frac{x}{|x|}
$$

which implies that $\Omega$ is a sphere.

In conclusion, a Maxwellian solution (A.2) to the Boltzmann equation (1.1) in a rotationally symmetric domain endowed with the specular reflection boundary condition reduces to the following form:

$$
\mu(x, v)=e^{c_{0}|v|^{2}+\varpi \times x \cdot v+a_{0}},
$$

where $\omega_{1}=\omega_{2}=0$ except for the case of $\Omega=\mathbb{S}^{3}$.

\section{ACKNOWLEDGEMENT}

Authors would like to thank Professor Yan Guo for fruitful discussions. The research of C, Kim was supported by FRG 07-57227. The research of S.-B. Yun was supported by the National Research Foundation of Korean Grant funded by the Korean Government (Ministry of Education, Science and Technology). [NRF-2010-357-C00005].

\section{REFERENCES}

1. Arkeryd, L., Cercignani, C.: A global existence theorem for the initial boundary value problem for the Boltzmann equation when the boundaries are not isothermal. Arch. Rational Mech. Anal. 125 (1993),no3, 271-288.

2. Arkeryd, L., Heinztz, A.: On the solvability and asymptotics of the Boltzmann equation in irregular domains. Comm. Partial Differ. Equ. 22 (1997) no. 11-12, 2129-2152.

3. Beals, R., Rrotopopescu, V.: Abstract time-dependent transport equations. J. Math. Anal. Appl. 212 (1987), no.2, 370-405.

4. Cercignani,C.: The Boltzmann equation and its applications, Springer, Berlin, 1988.

5. Cercignani,C., Illner, R., Pulvirenti, M.: The mathematical theory of dilute gases, Springer, Berlin, 1994.

6. Deimling, K.: Nonlinear Functional Analysis. Springer, Berlin, 1988.

7. Desvillettes, Convergence to equilibrium in large time for Boltzmann and B.G.K. equations Arch. Rational Mech. Anal. 110 (1990), no. 1, 73-91.

8. Diperna, R., Lions, P.-L.: On the Cauchy problem for the Boltzmann equation. Ann. Math. 130 (1989), no.2, 321-366.

9. Diperna, R., Lions, P.-L.: Global weak solution of Vlasov-Maxwell systems. Comm. Pure Appl. Math. 42 (1989), no.6, 729-757.

10. R. Glassey: The Cauchy problem in kinetic theory, SIAM, Philadelphia, 1996.

11. Y. Guo: Bounded solutions for the Boltzmann equation. Quart. Appl. Math. 68 (2000), no.1, 148-160.

12. Y. Guo: Continuity and Decay of the Boltzmann equation in bounded domains. Arch. Raional Mech. Anal. 197 (2010) no.3, 713-809 .

13. Y. Guo: The Vlasov-Poisson-Boltzmann system near Maxwellians. Comm. Pure Appl. Math. 55 (2002) no.9,1104-1135.

14. Y. Guo: The Vlasov-Maxwell-Boltzmann system near Maxwellians. Invent. Math. 153 (2003), no.3, 593630.

15. Y. Guo: Singular solutions of the Vlasov-Maxwell system on a half line. Arch. Ration Mech. Anal. 131 (1995), no.3, 241-304.

16. Y. Guo: Regularity for the Vlasov equations in a half-space. Indiana Univ. Math. J. 43 (1994), no.1, 255-320.

17. Guiraud, J.P.: An H-theorem for a gas of rigid spheres in a bounded domain, In: Pichon, G. (ed) Theories cinetique classique et relativistes, CNRS, Paris, pp.29-58, 1975.

18. Hamdache, K.: Initial boundary value problems for Boltzmann equation. Global existence of week solutions. Arch. Ration Mech. Anal. 119 (1992), 309-353.

19. Hwang, H.-J.:Regularity for the Vlasov-Poisson system in a convex domain. SIAM J. Math. Anal. 36 (2004), no. 1, 121-171. 
20. Hwang, H.-J.: Velazquez, J.: Global existence for the Vlasov-Poisson system in bounded domain. Arch. Rational Mech. Anal. 195 (2010), no. 3, 763-796.

21. Kim, C.:Formation and propagation of discontinuity for Boltzmann equation in non-convex domains. Preprint. arXiv: 1007.1997.

22. Liu, T.-P., Yu, S.-H.: Boltzmann equation, boundary effects. Discrete Contin. Dyn. Syst. 24 (2009), no. $1,145-157$.

23. Liu, T.-P., Yu, S.-H.: Initial-boundary value problem for one-dimensional wave solutions of the Boltzmann equation. Comm. Pure Appl. Math. 60 (2007) (3), 295-356.

24. Liu, T.-P., Yu, S.-H.: Green's function of Boltzmann equation, 3-D waves. Bull. Inst. Math. Acad. Sin. (N.S.) 1 (2006), no. 1, 1-78

25. Liu, T.-P., Yu, S.-H.: The Green's function and large-time behavior of solutions for the one-dimensional Boltzmann equation. Comm. Pure Appl. Math. 57 (2004), no. 12, 1543-1608.

26. Maslova, N.B.: Nonlinear Evolution Equations. Kinetic Approach. World Scientific, Singapore, 1993.

27. Mischler, S.: On the initial boundary value problem for the Vlasov-Poisson-Boltzmann system. Commun. Math. Phys. 210 (2000),no. 2, 447-466.

28. Masmoudi, N., Saint-Raymond, L.: From the Boltzmann equation to the Stkoes-Fourier system in a bounded domain. Comm. Pure App. Math. 56 (2003), no. 9, 1263-1293

29. Shizuta, Y.: On the classical solutions of the Boltzmann equation. Comm. Pure Appl. Math. 36 (1983), no. 6, 705-754.

30. Shizuta, Y., Asano, K.: Global solutions of the Boltzmann equation in a bounded convex domain. Proc. Jpn. Acad. 53A (1977), 3-5.

31. Sone, Y.: Kinetic theory and fluid dynamics. Birkhauser, Boston, 2002.

32. Molecular gas dynamics: Theory, techniques, and applications. Birkhauser, Boston, 2006.

33. Strain, R., Guo. Y.: Exponential decay for soft potentials near Maxwellians. Arch. Ration Mech. Anal. 187 (2008), no. 2, 287-339.

34. Ukai, S. : On the existence of global solutions of mixed problem for non-linear Boltzmann equation. proc. japan Acad. 50 (1974), 179-184.

35. Ukai, S. : Solutions of the Boltzmann equation. In: Pattern and Waves-Qualitative Analysis of Nonlinear Differnetial Equations. pp. 37-96, 1986.

36. Ukai, S., Asano, K.: On the initial boundary value problem of the linarized Boltzmann equation in an exterior domain. Proc. Jpn. Acad. 56 (1980), 12-17 .

37. Villani, C.: A review of mathematical topics in collisional kinetic theory. Handbook of mathematical fluid dynamics, Vol. I, 71-305, Noth-Holland, Amsterdam, 2002.

38. Villani, C.: Hypocoercivity. Mem. Amer. Math. Soc. 202 (2009), no 950.

39. Vidav, I.: Spectra of perturbed semigroups with applications to transport thoery. J. Math. Anal. Appl. 30 (1970), 264-279.

Department of Mathematics, Brown University, Providence, Ri 02917, USA

E-mail address: ckim@math.brown.edu, ckim.pde@gmail.com

Division of Applied Mathematics, Brown University, Providence, Ri, 02812, USA

E-mail address: sbyun01@gmail.com, seokbae_yun@brown.edu 